\title{
Activation of Somatostatin Interneurons by Nicotinic Modulator Lypd6 Enhances Plasticity and Functional Recovery in the Adult Mouse Visual Cortex
}

\author{
${ }^{(1 D M a s a t o}$ Sadahiro, ${ }^{1,2,3,4,5}$ Michael P. Demars, ${ }^{1,2,3,4,5}$ Poromendro Burman, ${ }^{1,2,3,4,5}$ Priscilla Yevoo, ${ }^{1,2,3,4,5}$ \\ Andreas Zimmer, ${ }^{6}$ and ${ }^{1}$ Hirofumi Morishita ${ }^{1,2,3,4,5}$ \\ ${ }^{1}$ Department of Psychiatry, Icahn School of Medicine at Mount Sinai, New York, New York 10029, ${ }^{2}$ Department of Neuroscience, Icahn School of \\ Medicine at Mount Sinai, New York, New York 10029, ${ }^{3}$ Department of Ophthalmology, Icahn School of Medicine at Mount Sinai, New York, New \\ York 10029, ${ }^{4}$ Mindich Child Health and Development Institute, Icahn School of Medicine at Mount Sinai, New York, New York 10029, ${ }^{5}$ Friedman \\ Brain Institute, Icahn School of Medicine at Mount Sinai, New York, New York 10029, and ${ }^{6}$ Institute of Molecular Psychiatry, Medical Faculty, \\ University of Bonn, 53127 Bonn, Germany
}

The limitation of plasticity in the adult brain impedes functional recovery later in life from brain injury or disease. This pressing clinical issue may be resolved by enhancing plasticity in the adult brain. One strategy for triggering robust plasticity in adulthood is to reproduce one of the hallmark physiological events of experience-dependent plasticity observed during the juvenile critical period: to rapidly reduce the activity of parvalbumin (PV)-expressing interneurons and disinhibit local excitatory neurons. This may be achieved through the enhancement of local inhibitory inputs, particularly those of somatostatin (SST)-expressing interneurons. However, to date the means for manipulating SST interneurons for enhancing cortical plasticity in the adult brain are not known. We show that SST interneuron-selective overexpression of Lypd6, an endogenous nicotinic signaling modulator, enhances ocular dominance plasticity in the adult primary visual cortex (V1). Lypd6 overexpression mediates a rapid experiencedependent increase in the visually evoked activity of SST interneurons as well as a simultaneous reduction in PV interneuron activity and disinhibition of excitatory neurons. Recapitulating this transient activation of SST interneurons using chemogenetics similarly enhanced V1 plasticity. Notably, we show that SST-selective Lypd6 overexpression restores visual acuity in amblyopic mice that underwent early long-term monocular deprivation. Our data in both male and female mice reveal selective modulation of SST interneurons and a putative downstream circuit mechanism as an effective method for enhancing experience-dependent cortical plasticity as well as functional recovery in adulthood.

Key words: critical period; Lypd6; nicotinic; parvalbumin; plasticity; somatostatin

\section{Significance Statement}

The decline of cortical plasticity after closure of juvenile critical period consolidates neural circuits and behavior, but this limits functional recovery from brain diseases and dysfunctions in later life. Here we show that activation of cortical somatostatin (SST) interneurons by Lypd6, an endogenous modulator of nicotinic acetylcholine receptors, enhances experience-dependent plasticity and recovery from amblyopia in adulthood. This manipulation triggers rapid reduction of PV interneuron activity and disinhibition of excitatory neurons, which are known hallmarks of cortical plasticity during juvenile critical periods. Our study demonstrates modulation of SST interneurons by Lypd6 to achieve robust levels of cortical plasticity in the adult brain and may provide promising targets for restoring brain function in the event of brain trauma or disease.

\section{Introduction}

Experience-dependent plasticity heightens during juvenile critical periods to dynamically shape brain function and behavior, then declines into adulthood to ensure stability. This poses a challenge for functional recovery in later life after brain injury or disease (Hensch, 2004; Knudsen, 2004). For instance, incongruous vision during early childhood results in amblyopia, a

Received June 8, 2019; revised Apr. 15, 2020; accepted Apr. 16, 2020.

Author contributions: M.S., M.P.D., and H.M. designed research; M.S., M.P.D., P.B., P.Y., and H.M. performed research; A.Z. contributed unpublished reagents/analytic tools; M.S., M.P.D., P.B., P.Y., and H.M. analyzed data; M.S., M.P.D., and H.M. wrote the paper. vision disorder marked by an enduring loss of visual acuity with limited treatment options in adulthood (Wiesel, 1982). Res-

This work was supported by National Eye Institute Grants R01-EY-024918, R01-EY-026053, and R21-EY026702 to H.M., and Grant F31-EY-028829 to M.S.; National Institute on Drug Abuse Grant T32-DA-007135 to M.P.D.; National Institute of Mental Health Grant T32-MH-096678 to M.S., and Grant R21-MH-106919 to H.M.; the Knights Templar Eye Foundation to H.M.; the March of Dimes to H.M.; the Whitehall Foundation to H.M.; and the Brain and Behavior Research Foundation to H.M. A.Z. is a member of the Excellence Cluster Immunosensation. We thank Dr. Klas Kullander (Uppsala University, Uppsala, Sweden), Dr. Yury Garkun, and Dr. Milo R. Smith (Icahn School of Medicine at Mount Sinai) for their comments; Dr. Daniel M. Brady and Dr. Kevin Guise for assisting data analysis; Dr. Ming-Hu Han (Icahn School of Medicine at Mount Sinai) for providing technical expertise on optogenetics; and Dr. Yasmin Hurd and Dr. Michael Michaelides (Icahn School of Medicine at Mount Sinai) for expertise on chemogenetics. 
toration of heightened plasticity in the adult brain is thought to support the restoration of function from such debilitating conditions (Hensch and Quinlan, 2018).

Ocular dominance (OD) plasticity, wherein a brief monocular visual deprivation in early life results in a robust shift of the responsiveness of the primary visual cortex (V1) from the deprived eye to the nondeprived eye, has been a long-standing model for understanding the fundamental regulatory mechanisms of critical periods and discovering novel targets for restoring heightened cortical plasticity in adulthood. Recent studies identified one of the key hallmarks of V1 plasticity during the critical period where a transient disinhibition of the excitatory cortical network occurs immediately following visual deprivation to rapidly restore cortical activity to levels before visual deprivation to initiate competitive plasticity. This disinhibition is mediated by a reduction of perisomatic inhibition by the parvalbumin (PV)-expressing GABAergic interneurons (Hengen et al., 2013; Kuhlman et al., 2013; Feese et al., 2018). Importantly, this rapid deprivation-induced reduction of PV interneuron activity and disinhibition of local excitatory neurons does not occur in adulthood (Kuhlman et al., 2013). Notably, artificially replicating this transient reduction in PV interneuron activity in adulthood through inhibitory chemogenetics robustly reactivates the juvenile form of OD plasticity marked by depression of deprived eye response (Kuhlman et al., 2013). This indicates that disinhibition of the excitatory network should be a fundamental aim for identifying molecular or circuit targets for enhancing experience-dependent cortical plasticity and developing therapeutic strategies for recovery from brain disorders such as amblyopia.

Controlled inhibition of cortical PV interneurons may potentially be achieved through enhancement of local inhibitory inputs, particularly from somatostatin (SST)-expressing GABAergic interneurons. SST interneurons inhibit PV interneurons twice as potently as they do local pyramidal excitatory neurons (Cottam et al., 2013), and the SST-PV disinhibitory microcircuit is well recognized across cortical functions (Hu et al., 2011; Hioki et al., 2013; Pfeffer et al., 2013; Xu et al., 2013, 2019; Kato et al., 2015; Sturgill and Isaacson, 2015; Yaeger et al., 2019). However, only until recently the potential for of SST interneurons in reactivating V1 plasticity in the adult brain remained underexplored (Tang et al., 2014; Davis et al., 2015; Fu et al., 2015). Neuromodulation of SST interneurons, particularly through cholinergic signaling (Jia et al., 2010; Leão et al., 2012; Zhao-Shea et al., 2013; Chen et al., 2015), may represent one potential means for enhancing plasticity in the adult brain. A recent study revealed that V1 SST interneurons during the critical period have heightened sensitivity to cholinergic modulation, and with visual activity this mediates fundamental mechanisms of cortical plasticity, including somatic disinhibition as well as branch-specific dendritic inhibition of pyramidal neurons (Yaeger et al., 2019). While this sensitivity declines in adulthood, the cited study found that optogenetic stimulation of SST interneurons, concurrent with arousal, reactivates somatic disinhibition and branch-specific dendritic inhibition. While there are no known molecular targets in SST interneurons in the adult brain for enhancing cortical plasticity, perhaps selective

The authors declare no competing financial interests.

Correspondence should be addressed to Hirofumi Morishita at hirofumi.morishita@mssm.edu.

https://doi.org/10.1523/JNEUROSCI.1373-19.2020

Copyright $(\odot) 2020$ the authors enhancement of cholinergic modulation in V1 SST interneurons may be one strategy.

Our recent study revealed that Lypd6, an endogenous modulator of nicotinic acetylcholine receptors (nAChRs), is highly enriched in V1 SST interneurons (Demars and Morishita, 2014). The Ly6 family of proteins that includes Lypd6 are glycophosphatidylinositol (GPI) anchored to the membrane, share unique toxin-like structures, and are known to bind to the extracellular face of nAChRs and cell autonomously regulate their signaling (Miwa et al., 2012). Lypd6 is also known to potentiate calcium currents through nAChRs (Darvas et al., 2009). In this study, we sought to elucidate the potential for targeting nicotinic modulation through Lypd6 in SST interneurons in enhancing plasticity and functional recovery in adulthood.

\section{Materials and Methods}

Animals. All mice were housed in groups of two to five together with littermates of the same sex in standard and uniform cage sizes $(199 \times 391$ $\times 160 \mathrm{~mm}$ : width $\times$ depth $\times$ height; GM500, Tecniplast) under a $12 \mathrm{~h}$ light/dark cycle (lights on at 7:00 A.M., lights off at 7:00 P.M.) in a facility with constant temperature $\left(23^{\circ} \mathrm{C}\right)$ and ad libitum access to food and water. Wild-type (WT) C57BL/6 mice were obtained from The Jackson Laboratory and Charles River Laboratories. Both males and females were used. SST-IRES-Cre (SST-Cre; catalog \#013044, The Jackson Laboratory) was purchased and bred in-house. Bigenic lines were created through targeted breeding of above strains. Lypd6Tg mice were originally generated by A.Z. (Darvas et al., 2009) and transferred to H.M., and back-crossed to C57BL/6. All animal protocols were approved by the Institutional Care and Use Committee at Icahn School of Medicine at Mount Sinai.

Monocular deprivation. Monocular deprivation (MD) procedures for animals that underwent $4 \mathrm{~d}$ of MD (4d MD), $1 \mathrm{~d}$ of MD (1d MD), or long-term MD (LTMD) were conducted on the contralateral eye and with the ipsilateral eye left open. The mice were anesthetized with isoflurane during the entire procedure. Eyelid margins were trimmed using an iris scissor, and one eye was sutured closed for 1 or $4 \mathrm{~d}$. Following $\mathrm{MD}$, mice were returned to their home cage before extracellular recording and subsequent euthanasia. For both OD plasticity measurements (4d MD) and cell type-specific visually evoked firing rate assessments (1d MD), the deprived eye was reopened just before the start of recording. For amblyopic mice (LTMD), the deprived eye was opened after the closure of the V1 critical period [postnatal day 33 (P33)] and maintained to stay open until the day of visual evoked potential (VEP) recordings (P60).

Generation and validation of $A A V-L y p d 6$. Lypd6 was amplified from a cDNA library derived from whole mouse V1, subcloned into a pcDNA3.1(-) vector. Subclonings were performed using an isothermal DNA assembly method (Gibson Assembly, New England Biolabs) and transformed into Escherichia coli. Colonies with a correct insert were identified through DNA sequencing (GENEWIZ), cultured, and then isolated using a HiSpeed MidiPrep Kit (Qiagen). Expression was first examined by transfection of N2A cells in vitro. To create the pAAV vector, an inverted bicistronic $2 \mathrm{~A}$ sequence was inserted into pAAV-Efl $\alpha$ DIO-EGFP-WPRE-pA (catalog \#37084, Addgene) upstream of EGFP by PCR linearization and overhang production on pAAV vector. The pcDNA3.1(-)-Lypd6 vector was used as a template for the Lypd6 insert, which was subsequently inserted into the pAAV-DIO-EGFP-2A vector as described above to create a pAAV-DIO-EGFP-2A-Lypd6-WPRE-pA vector. After sequence verification, a large culture and Maxiprep isolation produced a purified vector that was sent to the Vector Core at the University of North Carolina at Chapel Hill (UNC Vector Core) for viral packaging using an AAV8 serotype. To confirm viral efficiency, adenoassociated viruses (AAVs) were stereotaxically injected (see below for stereotaxic injection details) into V1 of SST-Cre mice. After perfusion, sections were labeled with rabbit anti-somatostatin antibodies (1:1000; Peninsula Laboratories) and the SST interneuron-specific GFP 
expression was confirmed. V1 was microdissected from an additional cohort of mice to assay the overexpression of Lypd6 through quantitative PCR (qPCR). RNA was isolated using an RNeasy Lipid Tissue Mini Kit (Qiagen) and cDNA was produced. The cDNA was subjected to qPCR analysis using a Life Technologies TaqMan assay (Thermo Fisher Scientific) at the Icahn School of Medicine at Mount Sinai Quantitative PCR CORE Facility.

Stereotaxic injection. Mice were isoflurane anesthetized and head fixed in a mouse stereotaxic apparatus (Narishige). The age range for surgery was P60 minimum to P78 maximum, with median age at P64. A midline incision was made in the scalp and a micro drill was used to drill a small hole in the skull over the right visual cortex. Three injections $(0.5 \mu \mathrm{l}$ each) were made into the deep layers of $\mathrm{V} 1$ binocular zone [from $\lambda$ : first injection: anteroposterior (AP), 0.0 ; mediolateral (ML), 3.1; dorsoventral (DV), 0.6; second injection: AP, 0.0; ML, 2.85; DV, 0.6; third injection: AP, 0.3 ; ML, 3.0; DV, 0.6] through glass micropipettes (diameter, $1.14 \mathrm{~mm}$; $\sim 20-30 \mu \mathrm{m}$ at opening) mounted to a Nanoject III Nanoliter Injector (Drummond Scientific) at $1 \mathrm{nl} / \mathrm{s}$. The syringe remained in place for 5 min following injection to reduce the backflow of virus. After injections, the skull hole was sealed using Kwik-Sil (World Precision Instruments), and the scalp was sutured. The mice recovered from anesthesia in an empty cage over a warming pad. Following recovery, mice were returned to their home cage where they remained for $>3$ weeks to allow for viral incorporation before any additional procedures or testing. The following viral constructs were used in this study: AAV-Lypd6 (AAV8-EF1 $\alpha$-DIO-EGFP-2A-Lypd6); AAV-GFP (AAV8-hSyn-DIOEGFP; UNC Vector Core); AAV-mCherry (AAV8-hSyn-DIO-mCherry; Addgene); AAV-CaMKII-Cre (AAV8-CamKIIa-mCherry-Cre; UNC Vector Core); AAV-GqDREADD (AAV8-hSyn-DIO-hM3D(Gq)-mCherry; UNC Vector Core); AAV-ChR2 (AAV1-Ef1 $\alpha$-dflox-hChR2(H134R)mCherry-WPRE-hGH; Penn Vector Core/Addgene); and AAV-hSyn-Cre (AAV8-hSyn-mCherry-Cre; UNC Vector Core).

Chemogenetic activation of $h M 3 d(G q)$ designer receptor exclusively activated by designer drugs. Clozapine- $N$-Oxide (CNO; Sigma-Aldrich), a normally inert compound that specifically activates designer receptor exclusively activated by designer drugs (DREADD) receptors, was prepared in $0.9 \%$ saline and injected intraperitoneally into adult SST-Cre (see Fig. $4 E-H$ ) at a concentration of $3 \mathrm{mg} / \mathrm{kg}$. CNO or saline was injected immediately following MD and again $12 \mathrm{~h}$ later for DREADDinduced activation of SST interneurons during the first day of MD following the previously validated protocol in mouse visual cortex (Kuhlman et al., 2013).

In vivo extracellular electrophysiology. Preparatory surgery leading to and recording itself was performed initially under Nembutal/chlorprothixene anesthesia and then maintained with isoflurane (Bukhari et al., 2015). For animals that underwent either 4 or $1 \mathrm{~d}$ of monocular deprivation, the deprived (contralateral) eye was reopened just before the start of recordings. Visually evoked single-unit activity was recorded in response to a high-contrast single bar, generated by ViSaGe Stimulus Generator (Cambridge Research Systems), which laterally traveled across the monitor in a $4 \mathrm{~s}$ interval followed by a $6 \mathrm{~s}$ interstimulus interval per trial. Using an eye patch, the stimulus was separately presented to the contralateral eye and then to the ipsilateral eye for at least 12 trials each. For each animal, 3-10 single units were recorded in each of the 4-6 vertical penetrations spaced evenly $(250 \mu \mathrm{m}$ intervals $)$ across mediolateral extent of V1 to map the monocular and binocular zones to avoid sampling bias. A linear 16-channel electrode was used to record each vertical penetration, which allowed recording from upper (channels $1 \sim 8$ ) and lower (channels 9 16) cortical layers at similar weights. The signal detected from the probe was amplified and thresholded, and unit sorted using an OmniPlex Neural Recording Data Acquisition System (Plexon). By comparing experimental conditions from seven of the experimental groups that were used in our data, we found that the fraction of neurons recorded from upper layers $(0.5235 \pm 0.02350)$ did not differ from that of neurons recorded from lower layers $(0.4765 \pm 0.02350 ; n=35$ animals; $p=0.3247$, Student's $t$ test for paired samples). A line crossing or "geometric" online sorting method was used to capture visually evoked responses as units. To ensure single-unit isolation, the waveforms of recorded units were further examined using Offline Sorter (Plexon). To analyze the electrophysiology data, a normalized OD index of a single neuron was computed by a custom-made MATLAB (MathWorks) script. This program generates peristimulus time histograms from each trial of visual stimulus presentation and uses a sliding window method to calculate the maximum response during stimulus presentation, and then baseline (spontaneous) spiking activity by bootstrapping with the same sized sliding window during prestimulus. The program then compares the mean maximum visually evoked response to the mean baseline spiking activity using a paired $t$ test to verify the significance of the visually evoked response. The analyzed peak to baseline spiking activity in response to each eye was then used to calculate OD scores using the following formula: [[Peak(ipsi) - baseline (ipsi)] - [Peak (contra) baseline(contra)]]/[[Peak (ipsi) - base line(ipsi)]+ [Peak(contra) baseline (contra)]]. OD scores were converted from the OD index using a 7 -point classification scheme, as follows: -1 to $-0.5=1 ;-0.5$ to $-0.3=2 ;-0.3$ to $-0.1=3 ;-0.1$ to $0.1=4 ; 0.1-0.3=5 ; 0.3-0.5=6$; and $0.5-1=7$. For each binocular zone, the contralateral bias index $(\mathrm{CBI})$ is calculated according to the following formula: $[(n 1-n 7)+2 / 3(n 2-$ $n 6)+1 / 3(n 3-n 5)+N] / 2 N$, where $N=$ total number of cells and $n x=$ number of cells corresponding to OD score of $x$.

In experiments where visually evoked firing rates of SST or PV interneurons were analyzed, a principal component analysis (PCA) clusterbased spike shape template-based sorting method was used. Visually evoked spikes assumed to be spikes originating from the same neuron grouped into visually identifiable subsets of waveforms to form clusters on 2D PCA space. Spike shape templates were selected by manually drawing selection contours around PCA clusters. The selected spikes were then automatically averaged and used to create templates, which were then used to sort incoming visually evoked spikes of similar shape and placement in PCA space into units. This online method allows defining multiple visually responsive units on a single channel of the linear 16-channel electrode, allowing recording up to 175 total cells in the V1 across approximately four to six sampling sessions. The number of putative PV interneurons or narrow-spiking (NS) cells recorded per sampling across all animals from all groups ranged between 11 and 46 cells. Total cells (all cell types included) recorded, the number ranged from 53 to 172 . In agreement with previously reported sorting methods (Niell and Stryker, 2008; Stark et al., 2013), the fractions of NS cells recorded were between approximately $17.4 \%$ and $24.2 \%$ of the total neurons recorded (SST-GFP 1d MD, 24.2\%; SST-Lypd6 1d MD, 18.9\%; SST-GFP no MD, 17.4\%; SST-Lypd6 no MD, 20.5\%).

For the experiment where SST interneurons were optogenetically tagged, a Cre-dependent virus-expressing channelrhodopsin-2 [ChR2; AAV1-Ef1 $\alpha$-dflox-hChR2(H134R)-mCherry-WPRE-hGH; Penn Vector Core] was injected into the V1 binocular zone of SST-Cre mice. The expression of channelrhodopsin in a cell type of interest allowed the use of optogenetic stimulation with a blue light as a search stimulus to simultaneously sort stimulus-locked responses from cell types of interest, and then record their visually evoked responses, by using an optic fibercoupled 16-channel linear silicone probe (NeuroNexus). This allowed accurate and high-throughput recordings of specific cell types, even against broad baseline noise and activities of other neuronal populations. Optogenetically tagged SST interneurons of the V1 binocular zone were identified using a $473 \mathrm{~nm}$ (blue) laser search stimulus emitted and delivered through the optic fiber coupled to the silicone probe and oriented immediately above the V1 cortical surface. After sorting for optogenetically responsive units (SST interneurons expressing ChR2), the optogenetic stimulus was switched off. Then a high-contrast single bar visual stimulus was presented to each eye to record the visually evoked responses (spike firing rate) of the sorted units. Analysis of visually evoked activities of sorted SST interneurons were restricted to only those recorded from the lower half of the 16-channel linear silicone probe to focus on the SST interneurons of lower layer IV, layer V, and layer VI. Recording from mice with less favorable conditions, including suboptimal health and prepped brain conditions, resulted in overall reduced visually evoked responses and subsequently reduced the number of recorded neurons because of increased difficulty in recording. Because we deemed that such animals would potentially misrepresent their 
groups, we established a measure of qualitative control by setting a quantitative threshold on the number of recorded neurons per mouse to exclude recordings that were suboptimal. All animals with $<10$ analyzable lower layer SST interneurons recorded were excluded from the analysis. The normalized firing rate (baseline subtracted from peak firing rate) of each lower layer SST interneuron, in response to contralateral eye (or deprived eye in animals in $1 \mathrm{~d}$ monocular deprivation groups) stimulation, was computed by first using a custom-made MATLAB script by generating a peristimulus time histogram-based analysis of peak and baseline spiking activity in response to visual stimulus. The peak visually evoked activity through contralateral/deprived eye stimulation was then subtracted by baseline spiking activity to obtain the normalized visually evoked firing rate.

To isolate visually evoked responses from NS neurons, visual stimulus responsive cells were distinguished offline as NS cells or regular-spiking (RS) neurons by means of spike width-based (trough-to-peak time) classification. The spike-width criterion for separating into NS and RS neurons was established by measuring the spike width of optogenetically tagged PV interneurons. PV interneurons were optogenetically tagged by injecting a Cre-dependent virus expressing channelrhodopsin-2 [AAV1-Ef1 $\alpha$-dflox-hChR2(H134R)-mCherry-WPRE-hGH; Penn Vector Core] into V1 binocular zone of PV-Cre mice. During recording, NS cells were first identified and sorted, using a $473 \mathrm{~nm}$ laser search stimulus delivered through the optical fiber coupled to the 16-channel silicone probe and oriented immediately above the V1 cortical surface, depending on their responsiveness to blue light stimulation within $3 \mathrm{~ms}$ post-blue light emission. The search stimulus was then exchanged to a visual stimulus. Visually evoked spike widths of the PV interneurons that were identified by optical tagging were then pooled to establish the official criterion for NS cells as having visually evoked spike width time (trough-to-peak time) of $<412 \mu$ s. The criterion was preliminarily tested in adult WT mice, and the NS neurons collected were confirmed as putative PV interneurons due to their similarly high visually evoked firing rate that was significantly higher compared with that of non-NS neurons. Averaged spike data for each unit were analyzed using a custom-made MATLAB script to obtain the spike width time for each unit. All NS cells sorted and recorded were included in the analysis of their visually evoked activity. Under the same basis applied to animals recorded for visually evoked responses in SST interneurons, all animals with $<10$ analyzable NS cells recorded were excluded. Using the same method for analyzing SST interneurons (one mouse from the SST-GFP 1d MD group and two mice from the SST-Lypd6 1d MD group), normalized firing rates of NS cells were calculated from responses to contralateral/deprived eye stimulation.

Following recordings, all AAV-injected mice were transcardially perfused, and the extent of GFP or mCherry signal was used to assess the viral transduction. Only mice that exhibited GFP or mCherry signal in the recorded V1 area were included for the analysis of OD plasticity or SST/ NS cell activity. The experimenters performed while blind to experimental conditions such as viral constructs or $\mathrm{CNO}$ /saline administration, but not monocular deprivation.

Visual evoked potential recording. Preparatory surgery leading to and recording itself was performed under Nembutal/chlorprothixene anesthesia and then maintained with isoflurane (Bukhari et al., 2015). A lowimpedance tungsten electrode (FHC) was inserted into the V1 binocular zone nearly $3.00 \mathrm{~mm}$ from $\lambda$, and to a depth between approximately 400 and $500 \mu \mathrm{m}$ from the pial surface, where maximal VEP amplitude can be measured in mice (Porciatti et al., 1999). Transient VEPs in response to horizontal sinusoidal gratings with abrupt contrast reversals (100\%, $1 \mathrm{~Hz}$ ) of varying spatial frequencies between approximately 0.05 and 0.5 cycles $/{ }^{\circ}$ were measured from signals that were bandpass filtered $(0.1 \sim 100 \mathrm{~Hz})$ and amplified (AM Systems), and were read using a custom software (National Instruments). VEP amplitudes from at least 30 events were averaged in synchrony with the stimulus contrast reversal. For each spatial frequency, between approximately four and five sessions of 30 events were sampled. Visual acuity was defined after VEP amplitudes were plotted against spatial frequency, and a nonlinear regression (semi-log) curve was applied to extrapolate the zero-amplitude point. The age range for VEP recordings was $\mathrm{P} 60$ minimum to $\mathrm{P} 74$ maximum with a median age at $\mathrm{P} 68$.
In situ hybridization. The production of probes and methodology for in situ hybridization has been previously described (Demars and Morishita, 2014). Briefly, RNA probes, including a fluorescein or digoxigenin (DIG) tag, were generated and used to label Lypd6, SST, vGlut1, GFP, and Chrna2 mRNA in $7 \mu \mathrm{m}$ sections of V1 from fresh frozen brains of animals at P28 (critical period: CP) and >P60 (adult). To fluorescently label mRNA, anti-fluorescein/DIG-peroxidase (POD; 1:2000; Roche) and anti-fluorescein/DIG-alkaline phosphatase (1:1000; Roche) antibodies were used. POD-conjugated antibody labeling of mRNA was proceeded with TSA Plus DNP signal amplification (PerkinElmer) and subsequent labeling with Life Technologies anti-DNP-KLH-488 antibodies (1:1000; Thermo Fisher Scientific). Alkaline phosphatase-conjugated antibody-labeled mRNA was stained using HNPP/Fast Red (Roche). Imaging was performed using an LSM780 Confocal Microscope (Zeiss). ImageJ was used to quantify the density of labeled pixels from each image or to examine colocalization using a color-based thresholding method. For the quantification of Lypd6 mRNA expression across age in V1, pixel density ( $>2$ SDs above mean intensity of the full image field) was determined from low-magnification images of V1 binocular zone using ImageJ software. For the quantification of colocalization of Lypd6 or SST with $n A C h R \alpha 2$, the number of cells positive for Lypd6/SST or $n A C h R \alpha 2$ in each image was determined using ImageJ by automated counting using a threshold of $>2$ SDs above background and limiting that to particles of $>40 \mu \mathrm{m}$ cell diameter. To calculate the colocalization percentage, the first color-based thresholding was used in Image to isolate and quantify the colocalized cells, then the percentage was calculated by dividing the number of colocalized cells by the number of $L y p d 6 / S S T$ - or $n A C h R \alpha 2$-positive cells in each image. For the quantification of colocalization of GFP and $v$ Glut1 mRNA, thresholding was first used to isolate and calculate the number of particles representing GFP-labeled cells ( $>40 \mu \mathrm{m}$ cell diameter). Then the corresponding $v$ Glut1-labeled image was redirected to the mask retained from the analysis of the GFP-labeled image to calculate the total number and then the percentage of GFP-labeled cells with colocalized $v$ Glut 1 labeling. For the quantification of Lypd6 mRNA expression in the V1 binocular zone of mice injected with a cocktail of AAV-Lypd6 and AAV-CaMKII-Cre, low-magnification images of binocular V1 from the injected or the corresponding naive hemisphere were analyzed for particles after thresholding. The absolute intensity for each particle was then measured as the product of mean intensity and the area of the particle. All absolute intensity values were summed to obtain total binocular V1 intensity, which was subsequently normalized by dividing by the area (in square micrometers) of the V1 binocular zone. The V1 binocular zone of each image was assessed using The Mouse Brain in Stereotaxic Coordinates (Franklin and Paxinos, 1997) as a reference.

Immunohistochemistry. Anesthetized mice were transcardially perfused with cold $4 \%$ paraformaldehyde (PFA) dissolved in $0.1 \mathrm{~m}$ phosphate buffer. The brains were postfixed in $4 \%$ PFA at $4^{\circ} \mathrm{C}$, and cryoprotected in $30 \%$ sucrose solution. The frozen brains were sectioned into $30-\mu \mathrm{m}$-thick coronal sections using a cryostat (model CM3050, Leica). Free-floating sections were washed in Tris-buffered saline (TBS), $\mathrm{pH}$ 7.5, and then blocked in $1 \%$ bovine serum albumin in TBST $(0.25 \%$ Triton X-100 in TBS) for $1 \mathrm{~h}$. The sections were incubated with mouse anti-parvalbumin (1:500; Swant) and rabbit anti-somatostatin (1:1000; Peninsula Laboratories). After primary antibody incubation, the slices were washed in TBST, followed by secondary antibody incubation with Alexa Fluor dyes (Thermo Fisher Scientific). Imaging was performed using a Zeiss LSM780 confocal microscope at $20 \times$ or $40 \times$ magnification. The V1 binocular zone of each image was assessed using The Mouse Brain in Stereotaxic Coordinates (Franklin and Paxinos, 1997) as a reference. The investigator performing the analysis was blind to the animal genotype.

Statistical analysis. The following statistical approaches were used for experiments assessing OD plasticity. A $\chi^{2}$ test was used to compare at cell level the distributions of OD scores between two groups and assess for the OD shift. For readability, the histogram figures for the distribution of OD scores represent the percentage of cells rather than the actual cell number. However, the $\chi^{2}$ statistics are the results of the tests 
A

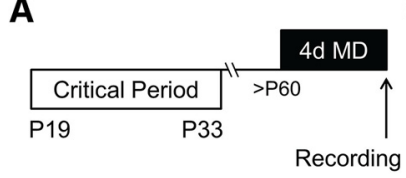

B
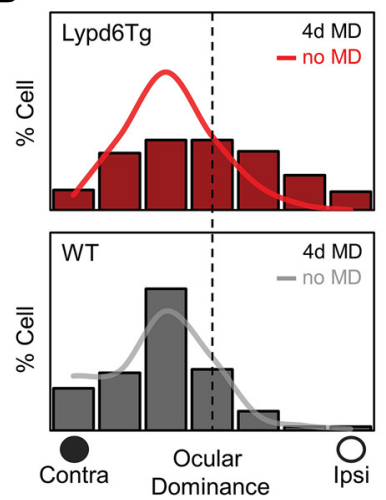

C

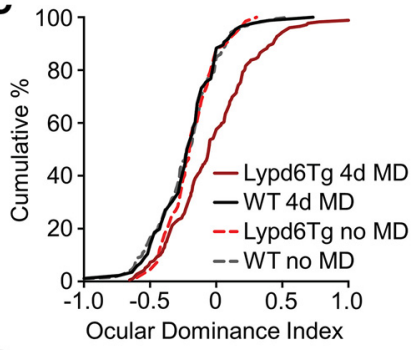

D

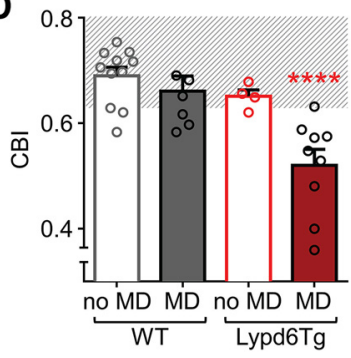

Figure 1. Neuronal overexpression of Lypd6 prolongs ocular dominance plasticity into adulthood. $\boldsymbol{A}$, Schematic of $\mathrm{V} 1$ plasticity paradigm with $4 \mathrm{~d} \mathrm{MD}$ ending with in vivo electrophysiological recording from the V1 binocular zone in adult ( $>$ P60) Lypd6Tg or WT mice. $\boldsymbol{B}$, Adult $4 \mathrm{~d}$ MD results in a shift in OD distribution of Lypd6Tg mice (red bar histogram; $n=$ 179 cells from 9 mice) but not of WT mice (gray bar histogram; $n=86$ cells from 7 mice), Lypd6Tg no MD (bright red line histogram; $n=67$ cells from 5 mice) and WT no MD (gray line histogram; $n=193$ cells from 11 mice). Statistics based on actual cell numbers recorded, but histograms represent percentage of cells with each OD scores. Main groups are presented in bar histogram format. Control groups are overlaid as line format. Filled circle labeled as "contra" represents contralateral eye that received monocular deprivation in 4d MD designated groups. Empty circle labeled as "ipsi" represents the ipsilateral nondeprived eye. $\boldsymbol{C}$, Cumulative plot of ODI after adult 4d MD confirms OD shift in Lypd6Tg mice [red line; $n=179$ cells from 9 mice] but not in WT mice (black line; $n=86$ cells from 7 mice), Lypd6Tg no MD (dashed bright red line; $n=67$ cells from 5 mice), and WT no MD (dashed gray line; $n=193$ cells from 11 mice). $D$, Comparison of (Bl following adult 4d MD in WT mice (gray solid bar; $\mathrm{CBI}=0.66, n=7$ mice) and Lypd6Tg mice (red solid bar; $\mathrm{CBI}=0.52$, $n=9$ mice), or no MD in WT mice (gray open bar; $(B \mid=0.66, n=11$ mice) and Lypd6Tg mice (red open bar; $(B I=0.65, n=5$ mice). Lypd6Tg $4 \mathrm{~d} M D$ mice have significantly decreased CBI values compared with all other groups (WT no MD, WT 4d MD, and Lypd6Tg no MD). Striped background area represents the (BI range of an adult WT mouse. Data are presented as the mean \pm SEM.

conducted on actual cell number. Cumulative distributions of OD index (ODI) were compared at cell level by using the Kolmogorov-Smirnov (K-S) test. One-way ANOVAwas used to compare CBI at animal levels, and Tukey's multiple-comparisons test was used for post hoc analyses. As justification of the use of a parametric test, the normality of the CBI data was tested by using the D'Agostino-Pearson test and Shapiro-Wilk test on any groups where $n$ is the number of mice exceeding the criteria for the tests ( $n>8$ and $n>7$, respectively). Normality was confirmed in all groups where tests were applicable, as follows: WT MD, WT no $\mathrm{MD}$, Lypd6Tg 4d MD, and SST-Lypd6 4d MD. In addition, we applied the Shapiro-Wilk test to the CBI data of a key experimental group from our previous study (Smith et al., 2016) that used the same experimental design and statistical analyses for testing OD plasticity and confirmed normality. Comparisons of NS cell (PV interneuron) and SST interneuron firing rates were conducted using one-way ANOVA with Tukey's multiple-comparisons test for post hoc analyses. A Student's $t$ test was used for all other analyses that compare mean differences between two groups. A minimum $p$ value of 0.05 was accepted as statistically significant. All statistical analyses were completed using Prism version 6.0 (GraphPad Software). All CBI, quantified expression, quantified colocalization, and visually evoked firing rate data in figures are presented as the mean \pm SEM. No method of randomization was used to determine the allocation of samples. No statistical methods were used to predetermine sample sizes. However, all cell-level and animal-level sample sizes for all experiments,

including quantified expression/colocalization experiments and in vivo extracellular electrophysiological experiments, were selected based on previously published studies.

Data availability. The data that support the findings of this study are available from the corresponding author (H.M.) on reasonable request.

\section{Results}

Neuronal overexpression of Lypd6 prolongs ocular dominance plasticity into adulthood

We began by testing whether Lypd6 can prolong a heightened level of OD plasticity in adulthood beyond the V1 critical period. Lypd6 is known to potentiate calcium transmission through the nicotinic acetylcholine receptors (nAChRs; Darvas et al., 2009) in contrast to Lynx1, a fellow Ly6 family member known to limit nicotinic cholinergic signaling (Ibañez-Tallon et al., 2002; Miwa et al., 2006) and OD plasticity in adulthood (Morishita et al., 2010; Sadahiro et al., 2016). Therefore, we tested whether overexpression of Lypd6 would prolong OD plasticity into adulthood by using a transgenic mouse line that pan-neuronally overexpress Lypd6 under the human synapsin promotor (Lypd6Tg; Darvas et al., 2009). We subjected adult Lypd6Tg mice to $4 \mathrm{~d}$ of monocular visual deprivation of the contralateral eye (MD), and then after the final day the deprived eye was reopened to assess OD in the V1 through in vivo extracellular electrophysiology under anesthesia (Gordon and Stryker, 1996; Bukhari et al., 2015; Fig. 1A). Each recorded V1 neuron was assigned an OD score based on the relative balance of the visually evoked firing rates of the neuron in response to visual stimuli independently presented to the contralateral and ipsilateral eye (Gordon and Stryker, 1996). Adult Lypd6Tg mice that underwent 4d MD displayed an overall significant shift in eye preference of the V1 (OD shift) away from the deprived contralateral eye and toward the nondeprived ipsilateral eye (Lypd6Tg 4d MD vs WT 4d MD: $\chi^{2}(6)=28.71$, $\left.{ }_{* * * *} p<0.0001\right)$. On the contrary, we did not detect OD shifts in adult Lypd6Tg mice that were not subjected to monocular visual deprivation (no MD; Lypd6Tg 4d MD vs Lypd6Tg no MD: $\left.\chi^{2}(6)=20.87,{ }^{* * *} p<0.0001\right)$ or in adult WT mice that typically do not display plasticity regardless of $4 \mathrm{~d}$ of visual deprivation (Lypd6Tg no MD vs WT no MD: $\chi^{2}(6)=7.354, p=0.28, \chi^{2}$ test; Fig. $1 B)$. Comparisons of cumulative distributions of ODI scores $(-1=$ most ipsilateral dominant; = equal $/$ binocular; $1=$ most contralateral dominant) for all recorded neurons from each group showed a significantly higher percentage of single neuronal responses that were ipsilateral dominant in the Lypd6Tg 4d MD mice (Lypd6Tg 4d MD vs WT 4d MD: ${ }^{* * * *} p<0.0001$; vs Lypd6Tg no MD: ${ }^{* *} p=0.0002$; Lypd6Tg no MD vs WT no MD: $p=0.2332$, K-S test; Fig. 1C). Last, individual animal-level comparisons of CBI scores, the relative strength to which the visually evoked activity of V1 neurons from the contralateral eye dominates over that from the ipsilateral eye, showed a significant decrease only in the Lypd6Tg 4d MD mice $\left(F_{(3,27)}=10.70\right.$, ${ }_{* * * *} p<0.0001$, one-way ANOVA; Lypd6Tg 4d MD vs WT no $\mathrm{MD},{ }^{* * *} p<0.0001$; vs WT $4 \mathrm{~d} \mathrm{MD},{ }^{* *} p=0.0023$; vs Lypd6Tg no $\mathrm{MD},{ }^{*} p=0.0208$, Tukey's multiple-comparisons test; Fig. 1D). Together, the results suggest that overexpression of Lypd6 prolongs OD plasticity into adulthood and highlight a novel role of Lypd6 as a positive regulator of cortical plasticity.

SST interneuron-selective Lypd6 overexpression reactivates ocular dominance plasticity in adulthood

While the prior experiment demonstrated the role of Lypd6 in prolonging OD plasticity into adulthood, the Lypd6 transgenic 
line cannot provide temporal, spatial, and cell type details regarding this effect. In our previous study, we found that in the V1 Lypd6 is enriched in deep layer SST interneurons, and also to a lesser extent in a subset of glutamatergic neurons (Demars and Morishita, 2014). To characterize the selective effects of Lypd6 overexpression in adulthood in specific V1 neuronal subpopulations, we developed a Cre-dependent AAV with a vector for overexpressing Lypd6 (AAV8-DIO-EGFP-2A-Lypd6: AAV-Lypd6). To achieve SST interneuron-specific overexpression, we injected AAV-Lypd6 into the binocular V1 of adult SST-Cre mice. Based on a previously implemented method (Koike et al., 2016; Pakan et al., 2016), we also overexpressed Lypd6 in V1 glutamatergic neurons of adult WT mice through viral cocktail injections of AAV-Lypd6 and a Cre expression AAV vector driven by a CaMKII promoter (AAV8-CamKIIa-mCherry-Cre: AAVCaMKII-Cre; Fig. 2A). The viral injections in SST-Cre mice were adjusted to target the deep V1 areas containing layers V and VI, where Lypd6 is endogenously expressed (Demars and Morishita, 2014; Fig. 2B). Both SST- and CaMKII-targeted injection strategies resulted in robust target-specific viral expression. A total of 98\% of GFP-labeled cells in adult SST-Cre mice were colabeled with SST, while $67 \pm 5 \%$ of SST-immunolabeled cells coexpressed viral GFP (Fig. 2C). A total of $93.2 \%$ of successfully cotransfected cells (GFP-positive as a result of both viral CaMKII-selective Cre and Cre-dependent Lypd6 expression) in adult WT mice were positive for the excitatory cell marker VGluT1, while $52.3 \pm 3 \%$ of VGluT1-positive cells coexpressed viral GFP (Fig. 2D). Overall, significantly increased Lypd6 mRNA was measured across the V1 in both SST-selective $\left(t_{(4)}=\right.$ 17.67, ${ }^{* * * *} p<0.0001$, Student's $t$ test) and CaMKII-selective Lypd6 overexpression $\left(t_{(4)}=3.742,{ }^{* *} p=0.0049\right.$, paired Student's $t$ test; Fig. 2E,F).

We assessed whether adult overexpression of Lypd6 in SST interneurons influences OD plasticity. We injected AAV-Lypd6 or control AAV vector (AAV8-hSyn-DIO-EGFP: AAV-GFP) into the V1 of adult SST-Cre mice and, after $>3$ weeks of viral incorporation, subjected them to $4 \mathrm{~d} \mathrm{MD}$. In adult mice with SST-specific Lypd6 overexpression, 4d MD (SST-Lypd6 4d MD) resulted in a significant OD shift (SST-Lypd6 4d MD vs CaMKII-Lypd6 4d MD: $\left.\chi^{2}(6)=29.72,{ }^{* * * *} p<0.0001\right)$. On the contrary, we did not detect OD shifts in nondeprived counterparts (vs SST-Lypd6 no MD: $\chi^{2}(6)=31.79,{ }^{* * * *} p<0.0001$ ) or control 4d MD mice that were injected with AAV-GFP (vs SSTGFP 4d MD: $\chi^{2}(6)=23.77,{ }^{* *} p=0.0006, \chi^{2}$ test; Fig. $\left.2 G\right)$. The cumulative distributions of ODI showed a significantly higher percentage of responses that were ipsilateral dominant only in the SST-Lypd6 4d MD mice (SST-Lypd6 4d MD vs CaMKIILypd6 4d MD, ${ }^{* * * *} p<0.0001$; vs SST-Lypd6 no MD, ${ }^{* * *} p=0.0001$; vs SST-GFP, ${ }^{* *} p=0.0002$, K-S test; Fig. $2 H$ ). Last, CBI was significantly decreased only in the SST-Lypd6 4d MD mice $\left(F_{(5,25)}=5.732,{ }^{* *} p=0.0012\right.$, one-way ANOVA; SST-Lypd6 $4 \mathrm{~d}$ MD vs SST-Lypd6 no MD, ${ }^{* *} p=0.0477$; vs SST-GFP 4d MD, ${ }^{* *} p=0.0183$; vs CaMKII-Lypd6 4d MD, ${ }^{* * *} p=0.0073$, Tukey's multiple-comparisons test; Fig. $2 I$ ). Because Lypd6 is also endogenously expressed in a subset of glutamatergic neurons, we also tested whether selective overexpression of Lypd6 in glutamatergic cells would induce plasticity in adult mice by injecting a viral cocktail of AAV-CaMKII-Cre and either AAV-Lypd6 or AAVGFP into V1 of adult WT mice. We did not observe a significant OD shift in mice with glutamatergic overexpression of Lypd6 that underwent 4d MD (CaMKII-Lypd6 4d MD; Fig. 2G). The distributions of neither OD scores nor $\mathrm{CBI}$ values were different from those of control counterparts (CaMKII-Lypd6 no MD and CaMKII-GFP 4d MD; Fig. 2H,I). Altogether, robust OD plasticity was measured only in the visually deprived adult mice that overexpressed Lypd6 specifically in SST interneurons. These results suggest that Lypd6 mediates reactivation of experiencedependent plasticity in adulthood through SST interneurons.

\section{Genetic deletion of $\alpha 2$ nicotinic acetylcholine receptor subunit abolishes Lypd6-mediated reactivation of ocular dominance plasticity}

We next aimed to determine the nAChR subtype required for reactivation of V1 plasticity mediated by Lypd6 in SST interneurons. Aside from the deep cortical layer subpopulation of SST interneurons (Demars and Morishita, 2014), Lypd6 is notably expressed in a subpopulation of hippocampal SST-positive oriens-lacunosum moleculare (O-LM) interneurons, which are physiologically similar to cortical SST-expressing Martinotti-type interneurons (Heys et al., 2012). O-LM interneurons are also distinguished by the expression of the $\alpha 2$-subunit-containing $\mathrm{nAChR}(\mathrm{nAChR} \alpha 2)$, the subtype that is most sparsely expressed across the brain and features unique nondesensitizing receptor kinetics (Jia et al., 2010; Demars and Morishita, 2014). In the cortex, the expression of $\mathrm{nAChR} \alpha 2$ is nearly exclusively limited to the layer V Martinotti-type SST interneurons (Ishii et al., 2005; Tasic et al., 2016; Hilscher et al., 2017; Tasic et al., 2018). In situ hybridization for Lypd6 and Chrna2 mRNA revealed that $79 \%$ of nAChR $\alpha 2$-positive cells coexpress Lypd6, and $>53 \%$ of Lypd6positive cells coexpress nAChR $\alpha 2$ (Fig. 3A). Furthermore, while $23 \%$ of SST-positive cells coexpress nAChR $\alpha 2,95 \%$ of nAChR $\alpha 2$-positive cells coexpress SST (Fig. $3 B$ ). Consistent with recent single-cell transcriptomic studies in adult V1 (Tasic et al., 2016, 2018), no overlaps were observed between $\mathrm{nAChR} \alpha 2$ and vasoactive intestinal peptide (VIP) or VGluT1, the markers of vasoactive intestinal peptide-expressing interneurons and pyramidal cells (data not shown). Together, this confirms strong preferential coexpression of Lypd6 and $\mathrm{nAChR} \alpha 2$ in a deep cortical layer subpopulation of SST interneurons.

We investigated whether experience-dependent plasticity after SST interneuron-specific overexpression of Lypd6 in adult mice requires $\mathrm{nAChR} \alpha 2$. To accomplish this, we created a bigenic SST-Cre/Chrna2KO mouse line to allow SST-specific Cre-recombinase expression on a background of Chrna2 knockout (Chrna2KO: $\alpha 2 \mathrm{KO}$; Lotfipour et al., 2013). Adult Chrna2KO mice have SST expression level and number of SST-positive cells comparable to those of adult WT and SST-Cre mice (adult WT, SST-Cre, a2KO mice SST-positive cell numbers: $F_{(1.119,2.238)}=$ $0.6923, p=0.502$; SST immunopositivity: $F_{(2,24)}=0.2158, p=$ 0.8075 , one-way ANOVA; Fig. $3 C$ ). Therefore, if plasticity induced by SST-selective Lypd6 overexpression is absent in the bigenic mice, then it would suggest an association of Lypd6 and $n A C h R \alpha 2$ in the induction of plasticity, and not a disruption of the SST interneuron population due to the genetic ablation of Chrna2. We injected AAV-Lypd6 into V1 of adult SST-Cre/ $\alpha 2 \mathrm{KO}$ bigenic mice, allowed $>3$ weeks for viral incorporation, and assessed OD plasticity after $4 \mathrm{~d} \mathrm{MD}$ (Fig. 3D). Intriguingly, the loss of $\mathrm{nAChR} \alpha 2$ (SST-Lypd6/ $\alpha 2 \mathrm{KO} 4 \mathrm{~d}$ MD) eliminated the robust cortical plasticity induced through SST-specific Lypd6 overexpression. Unlike the adult SST-Lypd6 4d MD mice, adult SST-Lypd6/ $\alpha 2 \mathrm{KO} 4 \mathrm{~d} \mathrm{MD}$ mice as well as deprived and nondeprived adult $\alpha 2 \mathrm{KO}$ mice ( $\alpha 2 \mathrm{KO} 4 \mathrm{~d} \mathrm{MD}$ and $\alpha 2 \mathrm{KO}$ no MD) did not show significant shifts in OD (SST-Lypd6 4d MD vs SSTLypd6/ $\alpha 2$ KO 4d MD: $\chi^{2}(6)=28.44,{ }^{* * * *} p<0.0001$; vs $\alpha 2 \mathrm{KO} 4 \mathrm{~d}$ MD: $\chi^{2}(6)=41.49,{ }^{* * *} p<0.0001$; vs $\alpha 2 \mathrm{KO}$ no MD: $\chi^{2}(6)=$ 
A

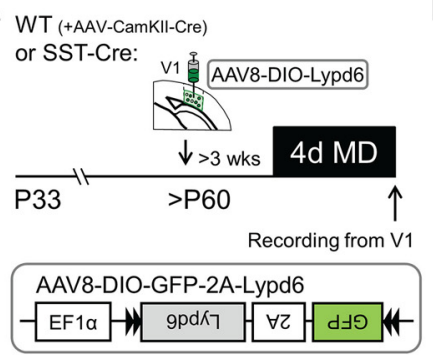

C
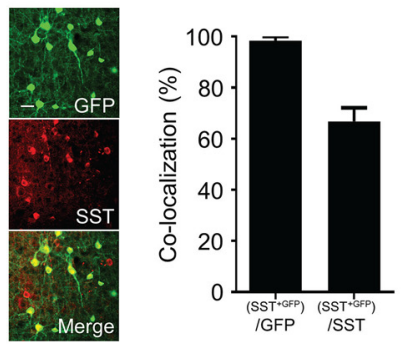

$\mathbf{E}$

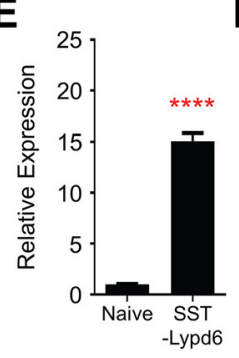

$\mathbf{F}$

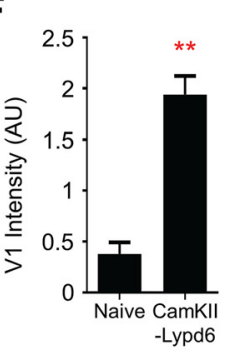

G

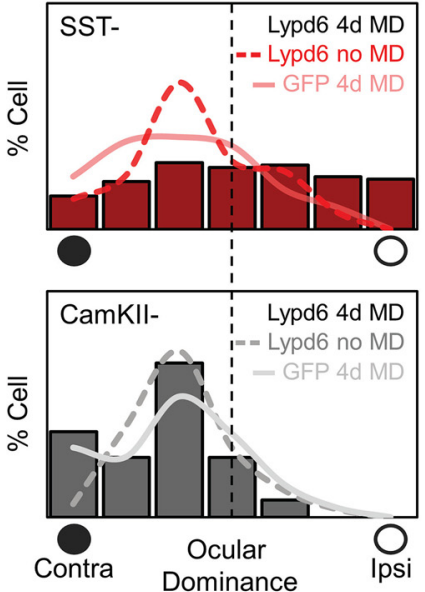

B

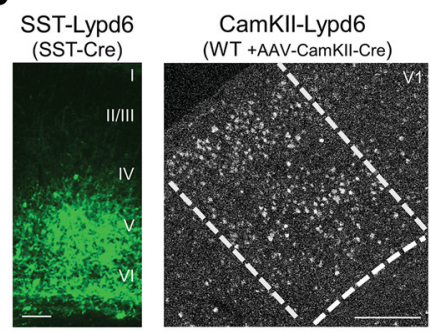

D

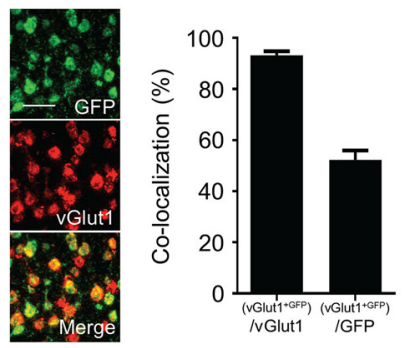

H
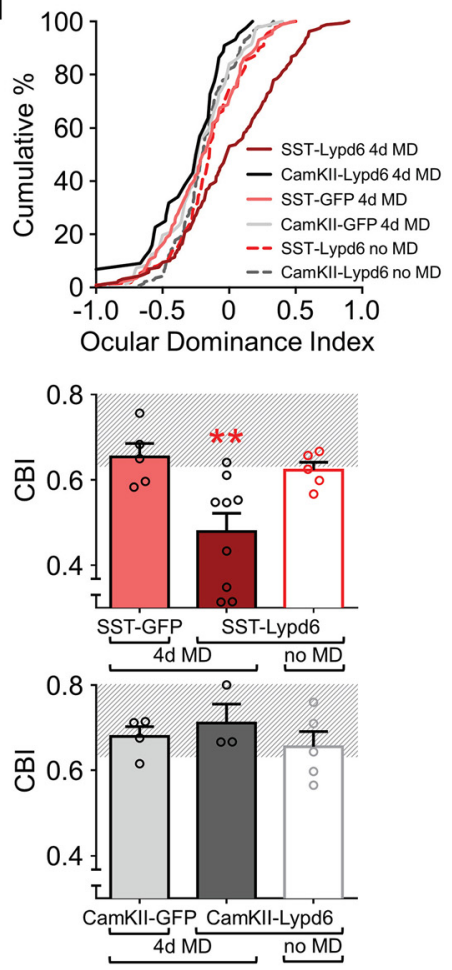

Figure 2. Selective overexpression of Lypd6 in SST interneurons reactivates ocular dominance plasticity in adult V1. $\boldsymbol{A}$, Schematic of 4d MD V1 plasticity paradigm with V1 stereotaxic injection of viral Lypd6 overexpression construct. AAV8-DI0GFP-2A-Lypd6 (see inset) was injected into the binocular zone of V1 of adult SST-cre mice or WT mice (coinjected with AAV(aMKII-cre) and incubated for $>3$ weeks before 4d MD. B, Left, Representative image of V1 following injection of AAV-Lypd6 in SST-Cre mice (SST-Lypd6), where viral transduction is represented by GFP (green). Scale bar, $100 \mu \mathrm{m}$. Right, Representative image of fluorescent in situ hybridization labeling of Lypd6 in V1 binocular zone following coinjection of AAV-Lypd6 and AAVCaMKII-Cre in WT mice (CaMKII-Lypd6). Scale bar, $200 \mu \mathrm{m}$. C, Representative immunohistochemistry in V1 binocular zone of SST-Lypd6 mouse showing viral GFP (green) overlapping immunolabeled SST (red) neurons. Scale bar, $50 \mu \mathrm{m}$. The $98 \pm 1.7 \%$ of cells expressing viral GFP were immunolabeled with SST. The $67 \pm 5 \%$ of SST-immunolabeled cells coexpressed viral GFP ( $n=2$ mice, 4 sections each, total from 8 sections: 190 colabeled cells, 194 GFP-labeled cells, 280 SST-labeled cells). D, Representative double in situ hybridization labeling of viral GFP in V1 binocular zone of a CaMKII-Lypd6 mouse showing specific expression of GFP (green) in VGluT1 (red)-positive neurons. Scale bar, $50 \mu \mathrm{m}$. The $93.2 \pm 1.6 \%$ of cells expressing GFP mRNA coexpress VGluT1 mRNA. The $52.3 \pm 3 \%$ of VGluT1 mRNA-positive cells coexpress viral GFP mRNA ( $n=3$ mice, 2 sections each, total from 6 sections: 979 colabeled cells, 1051 GFP-labeled cells, 1879 VGluT1-labeled cells). $\boldsymbol{E}$, Quantitative PCR of Lypd6 mRNA from cDNA derived from whole V1 extracts of naive (noninjected) and AAV-Lypd6-injected SST-cre mice ( $\triangle \Delta C T$ method, $n=3$ mice). $\boldsymbol{F}$, Quantification of Lypd6 expression in V1 binocular zone via fluorescent in situ-labeled sections from CaMKII-Lypd6 mice, compared between injected and naive (noninjected) hemispheres. (5 sections from 2 mice). $\mathbf{G}, 4 \mathrm{~d}$ MD in

48.51, ${ }^{* * *} p<0.0001, \chi^{2}$ test; Fig. $\left.3 E\right)$, shifts in cumulative distributions of ODI (SST-Lypd6 4d MD vs SST-Lypd6/ $\alpha 2 \mathrm{KO} 4 \mathrm{~d} \mathrm{MD},{ }^{* * *} p<0.0001$; vs $\alpha 2 \mathrm{KO}$ $4 \mathrm{~d} \mathrm{MD},{ }^{* * * *} p<0.0001$; vs $\alpha 2 \mathrm{KO}$ no MD, ${ }^{* * *} p<0.0001$, K-S test; Fig. $3 F$ ), or decreases in $\mathrm{CBI}\left(F_{(3,17)}=6.069,{ }^{* *} p=\right.$ 0.0053, one-way ANOVA; SST-Lypd6 4d $\mathrm{MD}$ vs SST-Lypd6/ $\alpha 2 \mathrm{KO} 4 \mathrm{~d} \mathrm{MD,}{ }^{* *} p=$ 0.0209 ; vs $\alpha 2 \mathrm{KO} 4 \mathrm{~d} \mathrm{MD},{ }^{* *} p=0.0450$; vs $\alpha 2 \mathrm{KO}$ no $\mathrm{MD},{ }^{* * *} p=0.0259$, Tukey's multiple-comparisons test; Fig. $3 G$ ). These findings suggest the requirement of $\mathrm{nAChR} \alpha 2$ in the induction of cortical plasticity by Lypd6 in SST interneurons.

\section{Rapid experience-dependent increase in SST interneuron activity drives ocular dominance plasticity under Lypd6 overexpression}

We next investigated the cell-autonomous impact of Lypd6 overexpression on SST interneuron activity that underlies reactivation of V1 plasticity. Engaging cholinergic signaling through Lypd6 overexpression may potentiate SST interneuron activity and in turn disinhibit excitatory neurons through inhibition of PV interneurons. This effect would match the hallmark event of ocular dominance plasticity during juvenile critical period wherein visual deprivation immediately results in a transient reduction of PV interneuron activity and in turn a disinhibition of the excitatory network (Hengen et al., 2013; Kuhlman et al., 2013; Feese et al., 2018). Considering Lypd6 is a positive modulator of nicotinic signaling (Darvas et al.,

SST-Lypd6 mice (SST-Lypd6 4d MD) results in OD shift (red bar histogram; $n=158$ cells from 9 mice) but not in CaMKII-Lypd6 mice (CaMKII-Lypd6 4d MD, gray bar histogram; $n=44$ cells from 3 mice), SST-Lypd6 no MD (dashed bright red line histogram; $n=110$ cells from 5 mice), and SST-GFP 4d MD (pink line histogram; $n=86$ cells from 5 mice). $\boldsymbol{H}$, Cumulative ODI plot confirms $0 D$ shift in SST-Lypd6 4d MD mice (red line; $n=158$ cells from 9 mice) but not in CaMKII-Lypd6 mice (black line; $n=44$ cells from 3 mice), SST-Lypd6 no MD (dashed bright red line; $n=110$ cells from 5 mice), and SST-GFP 4d MD (pink line; $n=86$ cells from 5 mice). I, Comparison of CBI following 4d MD in SST-Lypd6 (red solid bar; $(B I=0.48, n=9$ mice), SST-GFP (pink solid bar; $(B \mid=0.65, n=5$ mice), CaMKII-Lypd6 mice (dark gray solid bar; $(\mathrm{BI}=0.71, n=3$ mice), and (aMKII-GFP (light gray solid bar; $\mathrm{CBI}=0.68, n=4$ mice), with nondeprived SST-Lypd6 (red open bar; CBI $=0.62, n=5$ mice) and nondeprived CaMKII-Lypd6 (gray open bar; $(B \mathrm{I}=0.66, n=5$ mice). SST-Lypd6 4d MD significantly differs from SST-Lypd6 no MD, SST-GFP 4d MD, and CaMKII-Lypd6 4d MD. Data are presented as the mean \pm SEM. 
A

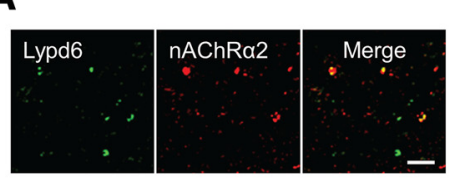

B

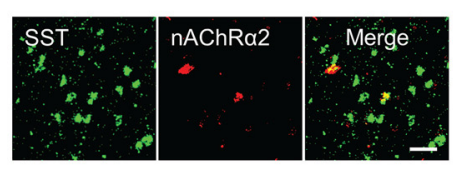

C

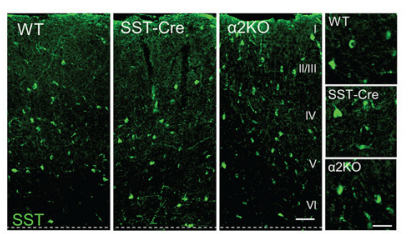

D

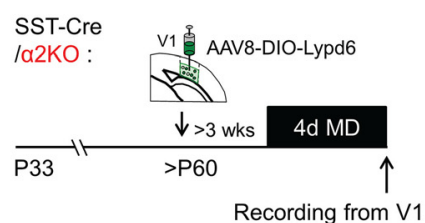

E

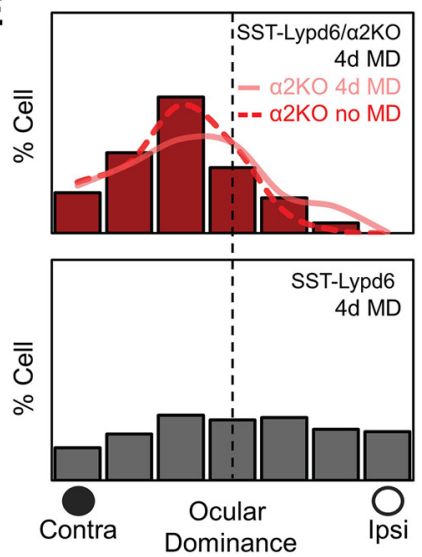

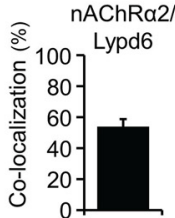
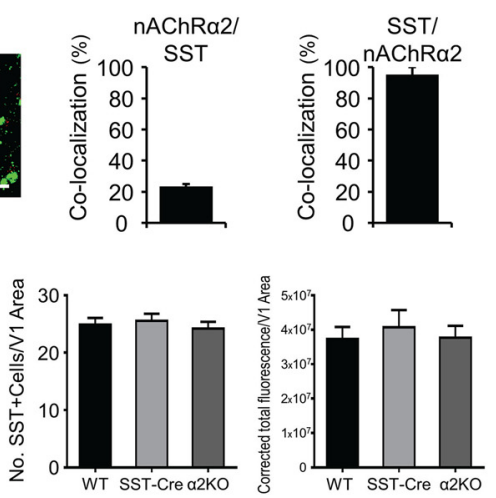

$\mathbf{F}$

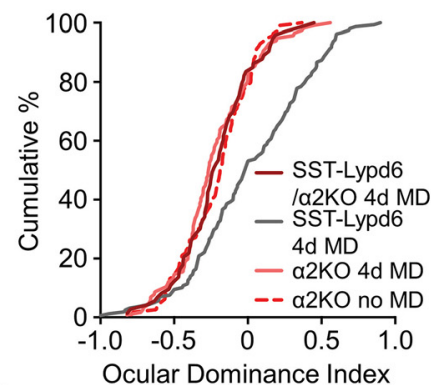

G

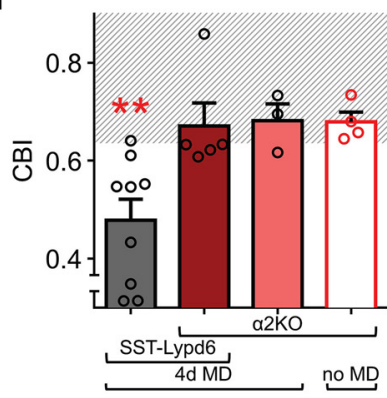

Figure 3. The $\alpha 2$ nicotinic acetylcholine receptor subunit is required for Lypd6-mediated reactivation of ocular dominance plasticity in adult V1. A, Double-fluorescent labeling of mRNA in layer V of adult V1 for Lypd6 (green) and $\alpha 2$ nicotinic acetylcholine receptor (Chrna2: nAChR $\alpha 2$; red). The $53 \pm 3.26 \%$ of Lypd6 mRNA-labeled cells coexpress nAChR $\alpha 2$. The $79 \pm 2.93 \%$ of nAChR $\alpha 2$ mRNA-labeled cells coexpress Lypd6 mRNA ( $n=2$ mice, 8 sections). B, Double-fluorescent in situ hybridization labeling of mRNA in layer V of V1 for SST (green) and $n A C h R \alpha 2$ (red). The $23 \pm 0.01 \%$ of SST mRNA-labeled cells coexpress nAChR $\alpha 2$ mRNA. The $95 \pm 0.04 \%$ of $n A C h R \alpha 2$ mRNA-labeled cells coexpress SST mRNA ( $n=2$ mice, 4 sections per mouse). Scale bars, $50 \mu \mathrm{m}$. C, Comparison of immunohistochemical labeling (green) of SST in V1 binocular zone of WT, SST-Cre, and Chrna2KO ( $\alpha 2 \mathrm{KO})$ mice. Scale bars: macro, $100 \mu \mathrm{m}$; close-up, $50 \mu \mathrm{m}$. Left graph, Number of SST + cells per V1 area. Right graph, Corrected total fluorescence per V1 area. WT, SST-Cre, and Chrna2KO mice have comparable total numbers of V1 somatostatin-positive cells or levels of somatostatin immunopositivity ( $n=3$ mice/group, 3 sections/mouse; group total: WT $=112$ cells, SST-Cre $=115$ cells, $\alpha 2 \mathrm{~K} 0=109$ cells). $D$, AAV-Lypd6 was injected into the V1 binocular zone of adult bigenic SST-cre/Chrna2KO mice (SST-Lypd6/ $\alpha 2$ KO) or adult SST-cre mice (SST-Lypd6) and was incubated for $>3$ weeks before 4d MD. E, OD shift is reduced in bigenic SST-Lypd6/ $\alpha 2 \mathrm{KO} 4 \mathrm{~d}$ MD mice (red bar histogram; $n=73$ cells from 5 mice) compared with SST-Lypd6 4d MD mice (gray bar histogram; data in Fig. 2G legend). 4d MD does not affect OD in adult Chrna2KO mice (pink line histogram; $n=116$ from 3 mice; $\alpha 2$ KO no MD, red dotted line histogram; $n=114$ from 4 mice). $\boldsymbol{F}$, Cumulative ODI plot confirms significantly reduced OD shift in the absence of $\mathrm{nAChR} \alpha 2$ (SST-Lypd6/ $\alpha 2 \mathrm{KO} 4 \mathrm{~d}$ MD; red line; $n=73$ cells from 5 mice) compared with SST-Lypd6 4d MD mice (gray line; data in Fig. $2 H$ legend). G, Comparison of CBI following 4d MD in SST-Lypd6 mice (gray solid bar; data in Fig. $2 /$ legend), SST-Lypd6/ $\alpha 2 \mathrm{KO}$ mice (red solid bar; $\mathrm{CBI}=0.66, n=5$ mice), and $\alpha 2 \mathrm{KO}$ mice (pink solid bar; $\mathrm{CBI}=0.68, n=3$ mice), or no MD in $\alpha 2 \mathrm{KO}$ mice (bright red open bar; $(B I=0.68, n=4$ mice). SST-Lypd6/ $\alpha 2 \mathrm{KO} 4 \mathrm{~d}$ MD is only significantly different with SST-Lypd6 4d MD. Data are presented as the mean \pm SEM.

2009), and SST interneurons are a significant source of inhibitory inputs to PV interneurons (Cottam et al., 2013; Hioki et al., 2013; Pfeffer et al., 2013), we hypothesized that rapid experience-dependent increase in the activity of SST interneurons drives OD plasticity achieved through adult SST-selective overexpression of Lypd6.

To evaluate how overexpression of Lypd6 impacts SST interneuron activity, we expressed ChR2 as an optogenetic tag to allow efficient identification of individual SST interneurons among a population of visually responsive units by using a blue light $(473 \mathrm{~nm})$ as a search stimulus. Using this technique, visually evoked single units were confirmed as SST interneurons if they also responded to optogenetic stimulation. We targeted the expression of ChR2 in SST interneurons by injecting an AAV with cre-dependent expression of ChR2 [AAV1-dflox-hChR2 (h134R)-mCherry-WPRE-hGH: AAVChR2) into the V1 of adult SST-Cre mice along with either AAV-Lypd6 (SSTLypd6) or AAV-GFP (SST-GFP). After $>3$ weeks of viral incorporation, the mice were subjected to just 1d MD (Fig. $4 A, B$ ). Considering the endogenous expression of Lypd6 is limited to deep cortical layers, we restricted our analyses to SST interneurons recorded from the lower half of the 16-channel linear silicone probe that correspond to layers IV-VI. There was a significant increase in the visually evoked firing rate of SST interneurons in adult SST-Lypd6 mice that underwent 1d MD (SST-Lypd6 1d MD), compared with 1d MD adult SST-GFP mice (SST-GFP 1d $\mathrm{MD})$ or nondeprived adult SST-Lypd6 (SST-Lypd6 no MD) mice $\left(F_{(3,267)}=\right.$ $13.45,{ }^{* * * *} p<0.0001$, one-way ANOVA; SST-Lypd6 1d MD vs SST-GFP 1d MD, ${ }^{* * * *} p<0.0001$; vs SST-GFP no MD, ${ }^{*} p=$ 0.02 ; and vs SST-Lypd6 no MD, ${ }^{* * *} p<$ 0.0001; Tukey's multiple-comparisons test; Fig. 4C,D). When baseline (spontaneous) firing rates without visual stimulation were compared, only a trending increase was observed when comparing SST-Lypd6 1d MD mice (4.47 spikes/s) and SST-GFP $1 \mathrm{~d}$ MD mice (2.87 spikes/ s), and no significant experience-dependent effects were observed SST-Lypd6 no MD (4.77 spikes/s; data not shown). This result suggests Lypd6 overexpression leads to a rapid experience-dependent increase in visually evoked SST interneuron activity.

To determine whether rapid increase in SST interneuron activity alone could mediate enhanced experience-dependent 
A

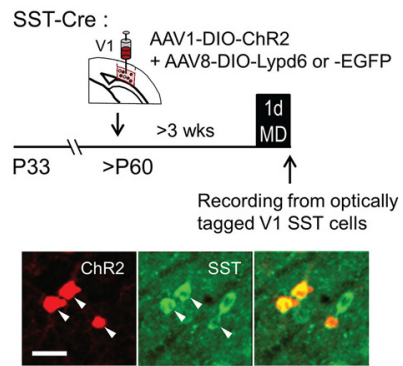

C

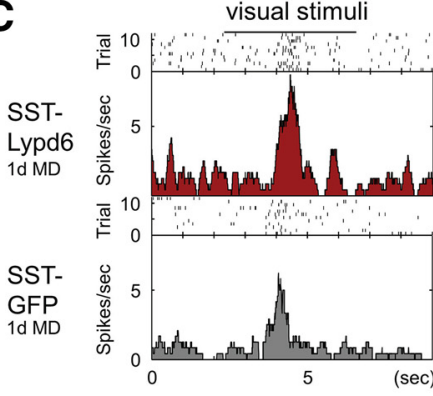

E

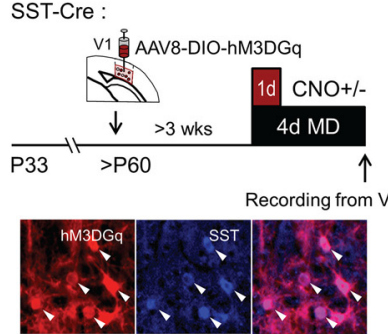

$\mathbf{F}$

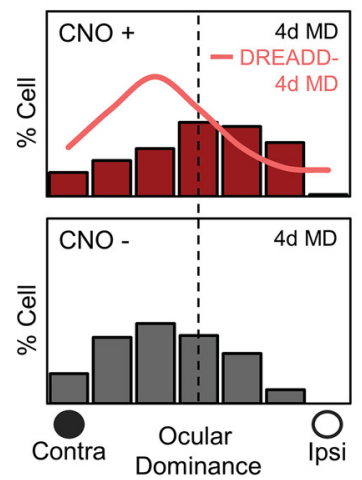

B
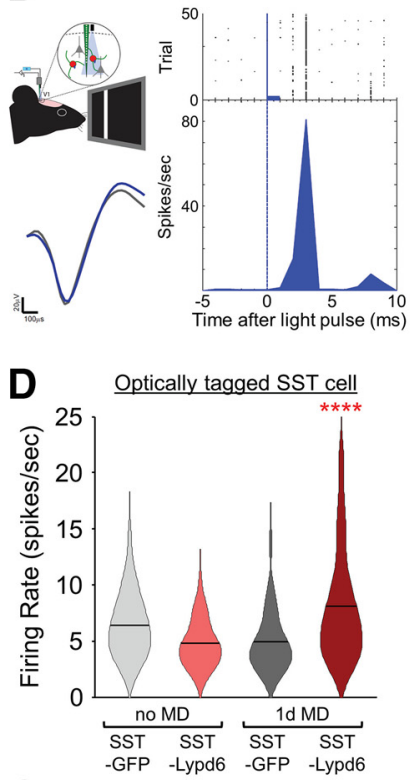

G

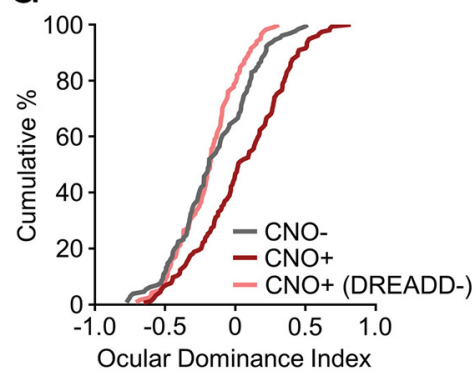

H

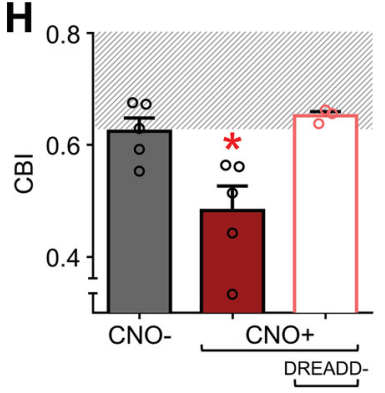

Figure 4. Lypd6 overexpression rapidly increases SST interneuron activity to express ocular dominance plasticity. $\boldsymbol{A}$, Adult SST-cre mice were injected with a combination of cre-dependent AAV-ChR2 and either AAV-Lypd6 (SST-Lypd6) or AAV-EGFP (SST-GFP) into the V1 binocular zone. Visually evoked firing rates of optically tagged SST interneurons were recorded after 1d MD. Bottom, Representative image showing viral ChR2/mCherry (red) overlapping immunolabeled SST (green) neurons. The $95 \pm 1.86 \%$ of ChR2/mCherry-labeled cells colabeled with SST ( $n=2$ mice, 177 of 187 cells; mean \pm SEM). Scale bar, 25 $\mu \mathrm{m}$. B, Top left, Schematic of in vivo electrophysiology setup with optical tagging. Bottom left, Representative traces of a putative SST interneuron from optical stimulation (blue trace) and averaged visually evoked spike (gray trace). Right, Histogram and raster plot shows time-locked optogenetic activation of optically tagged SST interneuron by $473 \mathrm{~nm}$ light pulses $(20 \mathrm{~Hz}$, $1 \mathrm{~ms}$ ) delivered through optic fiber positioned above the recording site and coupled to the multichannel linear electrode. $\boldsymbol{C}$, Representative histograms of visually evoked firing of optically tagged SST interneurons after 1d MD in SST-Lypd6 (red, top) and SST-GFP (gray, bottom). D, Comparison of visually evoked firing rate of SST interneurons in deep layer V1 following adult 1d MD in SST-GFP (gray solid bar; $n=68$ cells from 4 mice) and SST-Lypd6 mice (red solid bar; $n=101$ cells from 5 mice) with nondeprived (no MD) SST-GFP (gray open bar; $n=82$ cells from 5 mice), and SST-Lypd6 mice (red open bar; $n=38$ cells from 3 mice). 1d MD results in significant increase in visually evoked firing rate under Lypd6 overexpression (SST-Lypd6 1d MD) compared with controls: SST-GFP 1d MD, SST-GFP no MD, and SST-Lypd6 no MD. $\boldsymbol{E}$, AAV-GqDREADD was injected into V1 binocular zone of adult SST-cre mice and incubated for $>3$ weeks before $4 \mathrm{~d}$ MD. CNO (CNO ${ }^{+}$) or saline (CNO ${ }^{-}$) was given to only first day of $4 \mathrm{~d}$ MD. $\boldsymbol{F}$, Chemogenetic activation of SST interneurons during the first day of $4 \mathrm{~d} M D\left(C N 0^{+}:\right.$red, $n=154$ cells from 5 mice) results in significant OD shift, but not in mice given saline (CNO ${ }^{-}$: gray, $n=129$ cells from 5 mice) or 4d MD mice that received CNO without expressing DREADD (DREADD ${ }^{-}$: pink line histogram; $n=117$ cells from 3 mice). G, Cumulative ODI plot confirms OD shift after $4 \mathrm{~d} \mathrm{MD} \mathrm{in} \mathrm{CNO}^{+}$(red line; $n=154$ cells from 5 mice) compared with

plasticity in adulthood, we examined whether chemogenetic activation of SST interneurons in adult mice strictly during the first of the $4 \mathrm{~d}$ of monocular deprivation can reactivate OD plasticity (Fig. $4 E$ ). Adult SST-Cre mice were injected with an AAV with Cre-dependent expression of excitatory Gq protein-DREADD (AAV8hSyn-DIO-hM3D(Gq)-mCherry: AAVGqDREADD; Urban and Roth, 2015). Immediately following the start of $4 \mathrm{~d} \mathrm{MD}$, SST interneurons were activated for the first $24 \mathrm{~h}$ through the administration of CNO. As a result, the activation of SST interneurons in the adult V1 during the first of $4 \mathrm{~d}$ of monocular deprivation $\left(\mathrm{CNO}^{+}\right)$resulted in significant OD shift as opposed to control saline administered $\left(\mathrm{CNO}^{-}\right)$mice or mice given $\mathrm{CNO}$ but do not express DREADD receptors $\left(\mathrm{DREADD}^{-} ; \mathrm{CNO}^{+}\right.$vs $\mathrm{CNO}^{-}: \chi^{2}(12)=$ 53.12, ${ }^{* * *} p<0.0001$; vs DREADD ${ }^{-}$: $\chi^{2}(6)=42.52,{ }^{* * * *} p<0.0001, \chi^{2}$ test; Fig. $4 F$ ). The cumulative distributions of ODI show a significant shift in $\mathrm{CNO}^{+}$ mice and not in controls $\left(\mathrm{CNO}^{+}\right.$vs $\mathrm{CNO}^{-}:{ }^{* * *} p<0.0001$; vs DREADD ${ }^{-}$: ${ }^{* * * *} p<0.0001$, K-S test; Fig. 4G). Comparisons of CBI scores show a significant decrease only when SST interneurons were activated $\left(F_{(2,10)}=7.402\right.$, ${ }^{\star} p=0.0107$, one-way ANOVA; $\mathrm{CNO}^{+}$vs $\mathrm{CNO}^{-},{ }^{*} p=0.02$; vs DREADD ${ }^{-},{ }^{*} p=$ 0.02 , Tukey's multiple-comparisons test; Fig. 4H). Together, these results reveal a novel role for SST interneuron activity during the initial phase of visual deprivation in reactivating robust cortical plasticity in adulthood.

\section{SST interneuron-selective Lypd6 overexpression leads to rapid} experience-dependent suppression of $\mathrm{PV}$ interneuron and disinhibition of pyramidal neurons

After confirming immediate experiencedependent activation of SST interneurons underlies OD plasticity mediated by Lypd6 overexpression, we investigated how this affects downstream local circuits. We measured visually evoked activity of PV interneurons and excitatory neurons after $1 \mathrm{~d}$ of monocular deprivation under

CNO- (gray line; $n=129$ cells from 5 mice), or DREADD (pink line; $n=117$ cells from 3 mice). $\boldsymbol{H}$, Comparison of $(B I$ following $4 \mathrm{~d} \mathrm{MD} \mathrm{in} \mathrm{CNO}^{+}$(red solid bar; $\mathrm{CBI}=0.50, n=5$ mice), $\mathrm{CNO}^{-}$(gray solid bar; $\mathrm{CBI}=0.62, n=5$ mice), and DREADD $^{-}$(open pink bar; $\left(\mathrm{BI}=0.65, n=3\right.$ mice). $\mathrm{CNO}^{+}$ significantly differs from: $\mathrm{CNO}^{-}$and $\mathrm{DREADD}{ }^{-}$. Data are presented as the mean \pm SEM. 
A

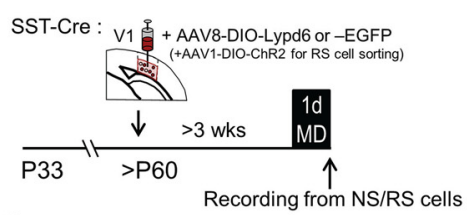

C
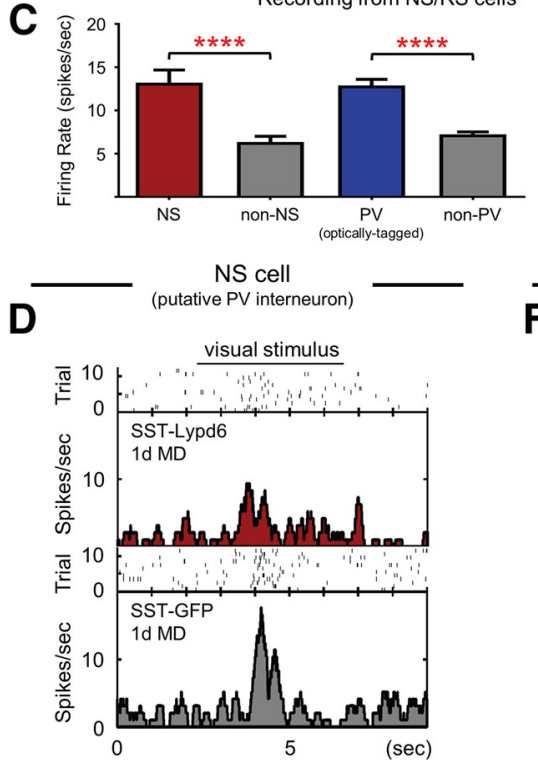

E

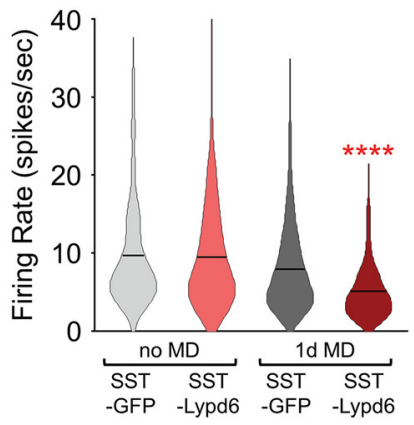

B

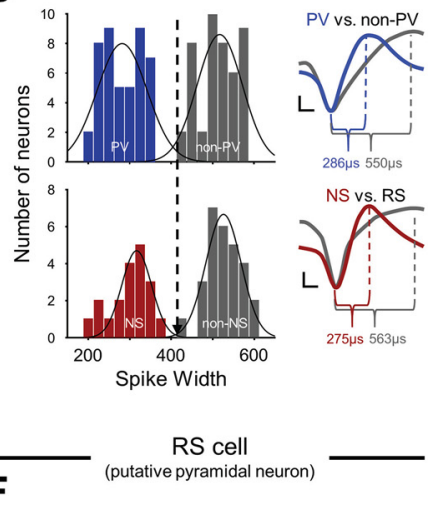

visual stimulus

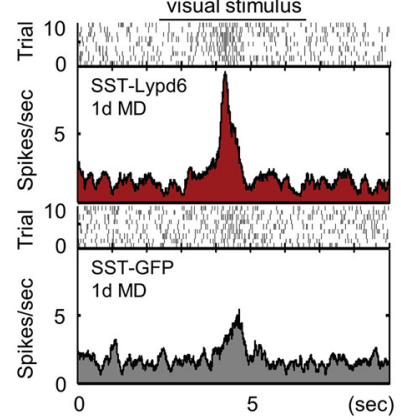

G

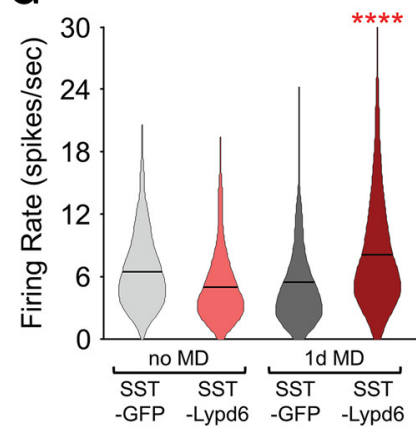

Figure 5. Rapid reduction in PV interneuron activity is required for ocular dominance plasticity under Lypd6 overexpression. $\boldsymbol{A}$, Adult SST-cre mice were injected with either AAV-Lypd6 (SST-Lypd6) or AAV-EGFP (SST-GFP) in the V1 binocular zone. For experiments involving RS cell (putative pyramidal neuron) sorting, AAV1-DI0-ChR2 was coinjected to exclude optically tagged SST interneurons. B, Criterion for assessing narrow-spiking units as putative PV interneurons (NS cells) is defined as neurons with spike width (trough-to-peak time) $<412 \mu$ s (dotted line), established from visually evoked spike widths of optically tagged (ChR2-expressing) PV interneurons in PV-cre mice [top histogram: PV (blue), 45 cells; non-PV (gray), 45 cells; 3 mice]. The criterion was then preliminarily applied to visual evoked spikes collected from adult WT mice [bottom histogram, NS (red), 19 cells; nonputative-PV interneuron (non-NS), 28 cells; 3 mice]. Right insets are representative traces of averaged visually evoked spikes from groups corresponding to the histograms (see details in Materials and Methods, In vivo extracellular electrophysiology). C, Sorted NS cells have higher average visually evoked firing rate than non-NS and non-PV cells, but are comparable to optically tagged PV cells. D, Representative histograms of visually evoked firing of NS cells after adult 1d MD in SST-Lypd6 (top, red) and SST-GFP (gray, bottom). $\boldsymbol{E}$, Comparison of visually evoked firing rate of NS cells following adult 1d MD in SST-GFP (gray solid bar; $n=148$ cells from 5 mice) and SST-Lypd6 (red solid bar; $n=170$ cells from 7 mice) with nondeprived (no MD) SST-GFP (gray open bar; $n=116$ cells from 5 mice) and SST-Lypd6 (red open bar; $n=63$ cells from 4 mice). 1d MD results in a significant decrease in the visually evoked firing rate of NS cells under SST-selective Lypd6 overexpression (SST-Lypd6 1d MD) compared with controls: SST-GFP 1d MD, SST-GFP no MD, and SST-Lypd6 no MD. $\boldsymbol{F}$, Representative histograms of visually evoked firing of RS cells after adult 1d MD in SST-Lypd6 (top, red) and SST-GFP (gray, bottom). G, Comparison of visually evoked firing rate of RS cells following adult 1d MD in SST-GFP (gray solid bar; $n=167$ cells from 5 mice) and SST-Lypd6 (red solid bar; $n=204$ cells from 6 mice) with nondeprived (no MD) SST-GFP (gray open bar; $n=240$ cells from 6 mice) and SST-Lypd6 (red open bar; $n=121$ cells from 4 mice). 1d MD results in a significant increase in visually evoked firing rate under Lypd6 overexpression (SST-Lypd6 1d MD) compared with controls: SSTGFP 1d MD, SST-GFP no MD, and SST-Lypd6 no MD. Data are presented as the mean \pm SEM.

SST-specific Lypd6 overexpression under the hypothesis that experience-dependent activation of SST interneurons through Lypd6 overexpression reduces PV interneuron activity and in turn disinhibits excitatory neurons. From adult SST-Cre mice injected with either AAV-Lypd6 (SST-Lypd6) or AAV-GFP (SST-GFP) and subjected to 1d MD (Fig. $5 A$ ), visually evoked cells were sorted based on spike width (trough-topeak time) into two categories. Putative PV neurons were defined by a narrow waveform that is electrophysiologically unique to PV interneurons. The criterion, or spike width cutoff of $412 \mu \mathrm{s}$, to determine NS neurons was established by collecting visually evoked spike widths of optically tagged $\mathrm{PV}$ interneurons in PV-Cre mice injected with AAV-ChR2 (Fig. 5B). The visually evoked firing rate of the NS neurons preliminarily sorted in WT mice via the criterion, were significantly higher than that of non-NS (nonputative PV) neurons and similar to that of optically tagged PV interneurons $\left(F_{(3,123)}=16.92^{* * * *} p<0.0001\right.$, one-way ANOVA; NS vs non-NS, ${ }^{* * *} p=0.0001$; vs non-PV, ${ }^{* * *} p=0.0001$; vs $\mathrm{PV}, p=0.9954$, Tukey's multiple-comparisons test; Fig. $5 C$; see Materials and Methods, In vivo extracellular electrophysiology). Additionally, pyramidal neurons were defined using a combination of spike width sorting and SST interneuron optical tagging to isolate nontagged and non-NS (RS) neurons.

In adult SST-Lypd6 mice that underwent $1 \mathrm{~d}$ of monocular deprivation of the contralateral eye (SST-Lypd6 1d MD), we found a significant reduction in visually evoked activity of NS neurons compared with all control groups, which suggests an experience-dependent decrease in the firing rate of NS neurons when Lypd6 is overexpressed in adult SST interneurons $\left(F_{(3,488)}=9.54,{ }^{* * *} p<0.0001\right.$, one-way ANOVA; SST-Lypd6 1d MD vs SST-GFP $1 \mathrm{~d} M D$, vs SST-GFP no MD, and vs SSTLypd6 no MD, all ${ }^{* * *} p<0.0001$, Tukey's multiple-comparisons test; Fig. 5D,E). Following that, we also found that only under SST-specific Lypd6 overexpression there is a significant experience-dependent increase in the visually evoked activity of non-SST RS neurons $\left(F_{(3,845)}=\right.$ 23.60, ${ }^{* * *} p<0.0001$, one-way ANOVA; SST-Lypd6 1d MD vs SST-GFP 1d MD, vs SST-GFP no MD, and vs SST-Lypd6 no MD, all ${ }^{* * * *} p<0.0001$, Tukey's multiple-comparisons test; Fig. 5F,G). The contrasting experience-dependent changes in visually evoked activity of NS neurons and RS neurons amid an increase in SST interneuron activity suggests that SST-specific Lypd6 overexpression may drive strong inhibition from SST to PV interneurons and in turn disinhibit the pyramidal neurons to rapidly fulfill the hallmark physiological event for initiating the cascade that results in reactivation of robust experience-dependent cortical plasticity in adulthood. 


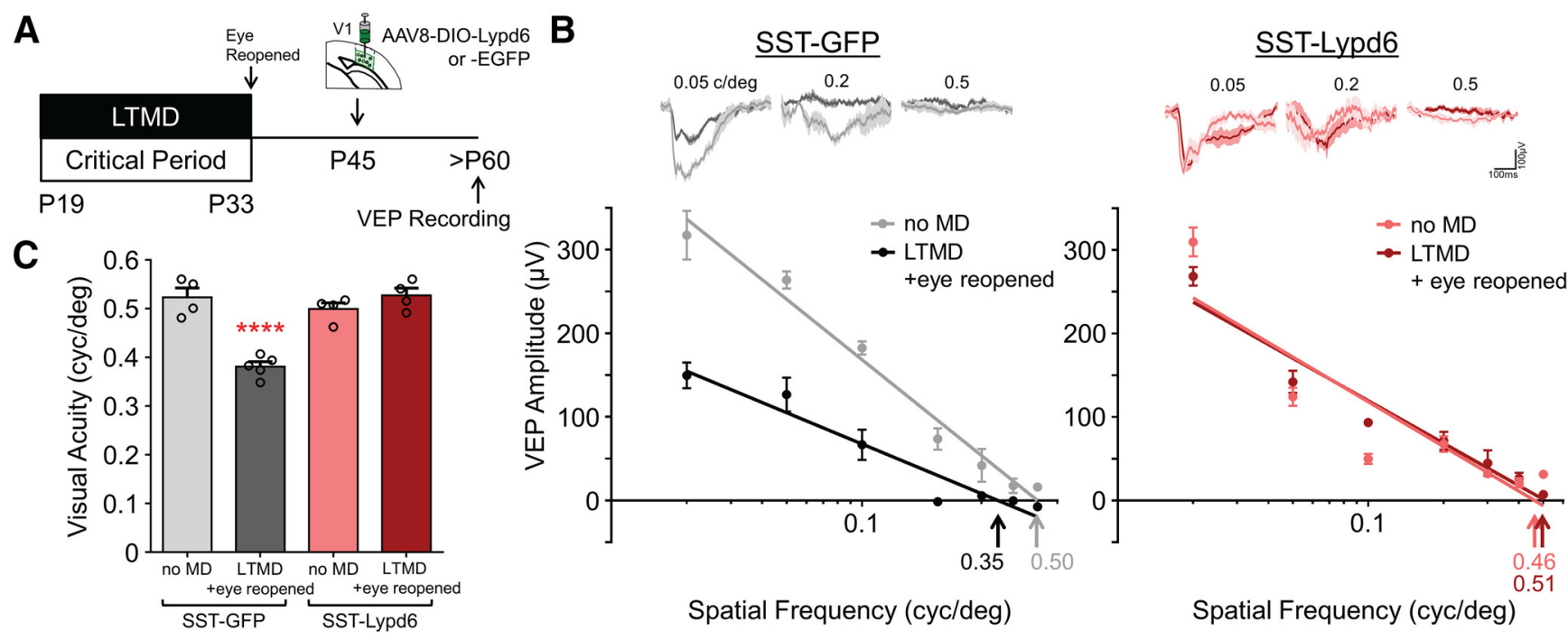

Figure 6. Functional recovery from amblyopia after V1 SST-selective overexpression of Lypd6. A, SST-cre mice were subjected to LTMD from P19 until P33, then the deprived eye was reopened, and binocular vision was maintained for the remainder. At P45, AAV-Lypd6 (SST-Lypd6) was injected into the V1 binocular zone. VEP was assessed after P60. B, Top, Representative VEP traces in response to sinusoidal grating stimuli at $0.05,0.2$, and 0.5 cycle ${ }^{\circ}$. Bottom, Representative plots of VEP amplitudes of first negative peaks measured from a series of spatial frequencies in adult SST-Cre mouse overexpressing either Lypd6 (right series) or control GFP (left series) and with or without LTMD (+ eye reopening): SST-GFP no MD (gray), SST-GFP LTMD+ eye opened (black), SST-Lypd6 no MD (pink), and SST-Lypd6 LTMD+eye reopened (red). Arrows indicate visual acuity determined by extrapolated spatial frequency at 0 V. C, Comparison of visual acuity following LTMD+eye opening in SST-GFP mice (dark gray; acuity $=0.3809, n=5$ mice) and SST-Lypd6 mice (red; acuity $=0.5315, n=4$ mice), or no MD in SST-GFP mice (light gray; acuity $=0.5229, n=4$ mice) and SST-Lypd6 mice (pink; acuity $=0.4990, n=4$ mice). SST-selective overexpression of Lypd6 post-LTMD restores visual acuity to levels comparable to those of nondeprived mice. Only SST-GFP LTMD + eye reopened shows significantly perturbed visual acuity, whereas SST-Lypd6 LTMD + eye reopened show visual acuity comparable to that of nondeprived controls. Data are presented as the mean \pm SEM.

SST interneuron-selective Lypd6 overexpression improves visual acuity after early long-term monocular deprivation Finally, we tested whether enhanced cortical plasticity induced through SST-selective overexpression of Lypd6 can promote robust functional changes in adulthood and investigated this in the context of recovering lost visual acuity in the mouse model of amblyopia. When wild-type mice are subjected to LTMD spanning the critical period, this results in persistent loss of visual acuity, which is measured directly in the V1 as the visual stimulus spatial frequency threshold that can evoke potential (VEP). Notably, visual acuity deficits endured after LTMD during the critical period cannot be recovered in adulthood even if the eye is reopened to restore and sustain visual input (Prusky and Douglas, 2003; Morishita et al., 2010). SST-Cre mice were subjected to LTMD between P19 and P33. Afterward, the deprived eye was reopened and maintained for the remainder of the experiment. At P45, the SST-Cre mice received V1 injections of either AAV-Lypd6 (SST-Lypd6) or control AAV-GFP (SSTGFP). Finally, after P60, the mice were tested for visual acuity of the previously deprived contralateral eye (Fig. 6A). Viral injections alone did not alter visual acuity, as all injected mice that were not subjected to early visual deprivation (no MD) had visual acuity that was comparable to that of adult SST-Cre mice naive to both visual deprivation and viral conditions (naive SSTCre mice average visual acuity, $0.51 \mathrm{cycles}^{\circ}$; data not shown in Fig. 6A). Among the mice that underwent LTMD followed by an eye opening, only the adult SST-GFP mice displayed expected enduring deficits in visual acuity. On the other hand, the visual acuity of the adult SST-Lypd6 mice that underwent LTMD and the subsequent eye reopening was significantly higher than that of the SST-GFP LTMD mice and was comparable to those of the nondeprived counterparts $\left(F_{(3,13)}=29.91,{ }^{* * * *} p<0.0001\right.$, oneway ANOVA; SST-GFP LTMD+eye reopened vs SST-GFP no $\mathrm{MD},{ }^{* * *} p<0.0001$; vs SST-Lypd6 no MD, ${ }^{* *} p=0.0001$; SST-
Lypd6 LTMD+eye reopened, ${ }^{* * * *} p<0.0001$, Tukey's multiplecomparisons test; Fig. $6 B, C$ ). The normal level of visual acuity, despite LTMD, after adult overexpression of Lypd6 selectively in V1 SST interneurons suggests recovery of visual function and the capacity of plasticity induced through Lypd6 or SST interneurons to drive restoration of cortical function that is typically beyond reach in adulthood.

\section{Discussion}

The decline of cortical plasticity after closure of the critical period is the key impedance of recovery from neurologic disorders and brain trauma in later life. The current study identifies a molecule and circuit mechanism that modulate SST interneurons in the adult V1 to achieve rapid experience-dependent cortical disinhibition, one of the hallmarks of juvenile critical period plasticity, to enhance OD plasticity. We find that Lypd6, an endogenous nAChR modulator, enhances experience-dependent plasticity through the activation of adult cortical SST interneurons and disinhibition of excitatory neurons through suppression of PV interneurons. Intriguingly, increased cortical plasticity by overexpression of Lypd6 in SST interneurons promoted recovery from amblyopia during adulthood. This study also provides bases for future studies to investigate the mechanistic action and subcellular localization of Lypd6 in SST interneurons. Furthermore, our findings may provide insight for the development of therapeutic compounds for treating brain disorders.

Like several of its fellow members of the Lynx family, Lypd6 is a GPI-bound membrane protein known to positively modulate nicotinic cholinergic signaling (Darvas et al., 2009; Miwa et al., 2012). Lypd6 may increase SST interneuron activity by impacting postsynaptic nicotinic modulation of excitatory synaptic inputs, such as in the case of hippocampal CA1 O-LM interneurons where $\mathrm{nAChR} \alpha 2$ gates LTP through receptor-mediated 
calcium influx (Jia et al., 2010). Alternatively, Lypd6 may modulate nicotinic signaling at presynaptic SST terminals to facilitate the release of GABA (Marchi and Grilli, 2010). Either potential mechanism can achieve the end goal of facilitation of SST interneuron activity. However, Lypd6 could mediate additional mechanisms that less directly influence nicotinic signaling. To date, the common subcellular localization of the Lynx family of proteins and the mechanism of nicotinic signaling modulation is largely unknown. However, evidence suggests that the modulatory mechanism of Lypd6 may be complex, that it may localize to synaptic compartments to also regulate ligand sensitivity or assembly and membrane insertion of nAChRs (Özhan et al., 2013; Nichols et al., 2014; Thomsen et al., 2014; Puddifoot et al., 2015; Arvaniti et al., 2016). Recently, a closely related Ly6 family protein known as Lypd6b was shown to modulate nAChRs through what seems to be counterintuitive actions, that it increases sensitivity for ACh, yet also reduces net current induced by ACh (Ochoa et al., 2016). Therefore, more mechanistic insight into the physiological action of Lypd6 on nicotinic signaling is eagerly awaited in future studies using new tools for selective targeting and manipulation.

Our study provides circuit-level insights into how selective modulation of SST interneurons can trigger rapid disinhibition for enhancing cortical plasticity in adulthood. We demonstrated that the overexpression of Lypd6 in SST interneurons rapidly leads to an experience-dependent increase in their visually evoked activity, and simultaneously a reduction in the visually evoked activity of PV interneurons and an increase in that of excitatory neurons. The suppression of PV interneuron activity and disinhibition of excitatory neurons are typically observed in juvenile mice after the first day of visual deprivation, and can trigger experience-dependent visual plasticity in adulthood when mimicked through chemogenetics (Kuhlman et al., 2013). To our knowledge, this is the first evidence that implicates the role of the SST-PV disinhibitory microcircuit in the adult cortex in enhancing cortical plasticity. Our findings suggest that an experience-dependent increase in SST interneuron activity not only impacts the spatiotemporal coordination of dendritic activity of pyramidal neurons (Silberberg and Markram, 2007; Cichon and Gan, 2015), but also increase the activity of pyramidal neurons potentially through suppression of PV interneurons. During the critical period, V1 SST interneurons have elevated sensitivity toward cholinergic signaling, which allows sensory experience and arousal to drive plasticity mechanisms including branch-specific dendritic activation and somatic disinhibition of excitatory neurons (Yaeger et al., 2019). The Lypd6 may perhaps be a key to reengaging cholinergic sensitivity in the SST interneurons of the adult cortex and restoring their circuit function for mediating robust and global levels of experience-dependent plasticity.

While the overexpression of Lypd6 in SST interneurons recapitulated rapid experience-dependent reduction of the PV interneuron activity and disinhibition of local excitatory neurons after $1 \mathrm{~d}$ of deprivation, it remains undetermined whether the subsequent enhanced OD plasticity after $4 \mathrm{~d}$ of MD is the juvenile form marked by the depression of deprived eye response, as it was in the case of adult mice that underwent chemogenetic suppression of PV interneurons during first day of MD (Kuhlman et al., 2013). Rather, our manipulation may have accelerated the initiation of the adult form of OD plasticity that typically takes approximately $7 \mathrm{~d}$ of $\mathrm{MD}$ that only results in the potentiation of the open eye and no change in deprived eye responses. Our approach to acutely measure contralateral biases of individual neurons through electrophysiology was not feasible to distinguish between deprived eye depression and open eye potentiation. Therefore, future investigation is warranted to test these alternative possibilities. Interestingly, a recent study showed that silencing SST interneurons during the entirety of $5 \mathrm{~d}$ of visual deprivation induces an adult form of OD plasticity, characterized by an elevation of open ipsilateral eye response ( $\mathrm{Fu}$ et al., 2015). Whether such a manipulation accompanies any form of disinhibition during the initial phase of visual deprivation is currently unknown. Their study and ours collectively highlight the capabilities of SST interneurons in engaging different forms of plasticity, perhaps dependent on the level of SST interneuron activity (reduced vs enhanced) and the timing of activity change (during the initial phase within $1 \mathrm{~d}$ vs later homeostatic phase after $5 \mathrm{~d}$ ). We propose a model where Lypd6 may function as a molecular switchboard for SST interneurons to shift modes and activate the SST interneurons to inhibit PV interneurons (Nakauchi et al., 2007; Leão et al., 2012) while disengaging the VIP-SST disinhibitory circuit that relieves inhibitory action of SST interneurons on the distal dendrites of pyramidal neurons (Chen et al., 2012; van Versendaal et al., 2012; Fu et al., 2015). In this model, both SST-PV and VIP-SST disinhibitory circuits can coexist to play complementary roles in the modulation of plasticity, depending on the context and brain state.

The capacity for nicotinic modulation by Lypd6 to induce robust experience-dependent cortical plasticity and functional recovery in the adult cortex opens the possibility of targeting this receptor to treat conditions such as amblyopia, stroke, and traumatic brain injury where hope for recovery in later life is low due to diminished plasticity. The selective expression of Lypd6 in deep-layer subpopulations of SST interneurons could limit offtarget effects (Timmermann et al., 2012; Wang et al., 2015a,b) and make them attractive as novel therapeutic targets, compared with interventions that do not regard subtype or receptor subunit specificity. Novel pharmacological interventions may be combined with behavioral interventions like physical exercise (Kalogeraki et al., 2014), which may further enhance the impact on SST interneuron activity (Polack et al., 2013; Fu et al., 2014; Reimers et al., 2014; Pakan et al., 2016; Dipoppa et al., 2018).

\section{References}

Arvaniti M, Jensen MM, Soni N, Wang H, Klein AB, Thiriet N, Pinborg LH, Muldoon PP, Wienecke J, Imad Damaj M, Kohlmeier KA, Gondré-Lewis MC, Mikkelsen JD, Thomsen MS (2016) Functional interaction between Lypd6 and nicotinic acetylcholine receptors. J Neurochem 138:806-820.

Bukhari N, Burman PN, Hussein A, Demars MP, Sadahiro M, Brady DM, Tsirka SE, Russo SJ, Morishita H (2015) Unmasking proteolytic activity for adult visual cortex plasticity by the removal of Lynx1. J Neurosci 35:12693-12702.

Chen JL, Villa KL, Cha JW, So PT, Kubota Y, Nedivi E (2012) Clustered dynamics of inhibitory synapses and dendritic spines in the adult neocortex. Neuron 74:361-373.

Chen N, Sugihara H, Sur M (2015) An acetylcholine-activated microcircuit drives temporal dynamics of cortical activity. Nat Neurosci 18:892-902.

Cichon J, Gan W-B (2015) Branch-specific dendritic $\mathrm{Ca}(2+)$ spikes cause persistent synaptic plasticity. Nature 520:180-185.

Cottam JC, Smith SL, Häusser M (2013) Target-specific effects of somatostatin-expressing interneurons on neocortical visual processing. J Neurosci 33:19567-19578.

Darvas M, Morsch M, Racz I, Ahmadi S, Swandulla D, Zimmer A (2009) Modulation of the $\mathrm{Ca} 2+$ conductance of nicotinic acetylcholine receptors by Lypd6. Eur Neuropsychopharmacol 19:670-681.

Davis MF, Figueroa Velez DX, Guevarra RP, Yang MC, Habeeb M, Carathedathu MC, Gandhi SP (2015) Inhibitory neuron transplantation 
into adult visual cortex creates a new critical period that rescues impaired vision. Neuron 86:1055-1066.

Demars MP, Morishita H (2014) Cortical parvalbumin and somatostatin GABA neurons express distinct endogenous modulators of nicotinic acetylcholine receptors. Mol Brain 7:75.

Dipoppa M, Ranson A, Krumin M, Pachitariu M, Carandini M, Harris KD (2018) Vision and locomotion shape the interactions between neuron types in mouse visual cortex. Neuron 98:602-615.e8.

Feese BD, Pafundo DE, Schmehl MN, Kuhlman SJ (2018) Binocular deprivation induces both age-dependent and age-independent forms of plasticity in parvalbumin inhibitory neuron visual response properties. J Neurophysiol 119:738-751.

Franklin KBJ, Paxinos G (1997) The mouse brain in stereotaxic coordinates. San Diego: Academic.

Fu Y, Tucciarone JM, Espinosa JS, Sheng N, Darcy DP, Nicoll RA, Huang ZJ, Stryker MP (2014) A cortical circuit for gain control by behavioral state. Cell 156:1139-1152.

Fu Y, Kaneko M, Tang Y, Alvarez-Buylla A, Stryker MP (2015) A cortical disinhibitory circuit for enhancing adult plasticity. Elife 4:e05558.

Gordon JA, Stryker MP (1996) Experience-dependent plasticity of binocular responses in the primary visual cortex of the mouse. J Neurosci 16:32743286.

Hengen KB, Lambo ME, Van Hooser SD, Katz DB, Turrigiano GG (2013) Firing rate homeostasis in visual cortex of freely behaving rodents. Neuron 80:335-342.

Hensch TK (2004) Critical period regulation. Annu Rev Neurosci 27:549579.

Hensch TK, Quinlan EM (2018) Critical periods in amblyopia. Vis Neurosci 35:E014.

Heys JG, Schultheiss NW, Shay CF, Tsuno Y, Hasselmo ME (2012) Effects of acetylcholine on neuronal properties in entorhinal cortex. Front Behav Neurosci 6:32.

Hilscher MM, Leão RN, Edwards SJ, Leão KE, Kullander K (2017) Chrna2Martinotti cells synchronize layer 5 type A pyramidal cells via rebound excitation. PLoS Biol 15:e2001392.

Hioki H, Okamoto S, Konno M, Kameda H, Sohn J, Kuramoto E, Fujiyama F, Kaneko T (2013) Cell type-specific inhibitory inputs to dendritic and somatic compartments of parvalbumin-expressing neocortical interneuron. J Neurosci 33:544-555.

Hu H, Ma Y, Agmon A (2011) Submillisecond firing synchrony between different subtypes of cortical interneurons connected chemically but not electrically. J Neurosci 31:3351-3361.

Ibañez-Tallon I, Miwa JM, Wang H-L, Adams NC, Crabtree GW, Sine SM, Heintz N (2002) Novel modulation of neuronal nicotinic acetylcholine receptors by association with the endogenous prototoxin lynx1. Neuron 33:893-903.

Ishii K, Wong JK, Sumikawa K (2005) Comparison of $\alpha 2$ nicotinic acetylcholine receptor subunit mRNA expression in the central nervous system of rats and mice. J Comp Neurol 493:241-260.

Jia Y, Yamazaki Y, Nakauchi S, Ito K, Sumikawa K (2010) Nicotine facilitates long-term potentiation induction in oriens-lacunosum moleculare cells via $\mathrm{Ca} 2+$ entry through non-alpha7 nicotinic acetylcholine receptors. Eur J Neurosci 31:463-476.

Kalogeraki E, Greifzu F, Haack F, Löwel S (2014) Voluntary physical exercise promotes ocular dominance plasticity in adult mouse primary visual cortex. J Neurosci 34:15476-15481.

Kato HK, Gillet SN, Isaacson JS (2015) Flexible sensory representations in auditory cortex driven by behavioral relevance. Neuron 88:1027-1039.

Knudsen EI (2004) Sensitive periods in the development of the brain and behavior. J Cogn Neurosci 16:1412-1425.

Koike H, Demars MP, Short JA, Nabel EM, Akbarian S, Baxter MG, Morishita H (2016) Chemogenetic inactivation of dorsal anterior cingulate cortex neurons disrupts attentional behavior in mouse. Neuropsychopharmacology 41:1014-1023.

Kuhlman SJ, Olivas ND, Tring E, Ikrar T, Xu X, Trachtenberg JT (2013) A disinhibitory microcircuit initiates critical-period plasticity in the visual cortex. Nature 501:543-546.

Leão RN, Mikulovic S, Leão KE, Munguba $\mathrm{H}$, Gezelius H, Enjin A, Patra K, Eriksson A, Loew LM, Tort AB, Kullander K (2012) OLM interneurons differentially modulate CA3 and entorhinal inputs to hippocampal CA1 neurons. Nat Neurosci 15:1524-1530.
Lotfipour S, Byun JS, Leach P, Fowler CD, Murphy NP, Kenny PJ, Gould TJ, Boulter J (2013) Targeted deletion of the mouse $\alpha 2$ nicotinic acetylcholine receptor subunit gene (Chrna2) potentiates nicotine-modulated behaviors. J Neurosci 33:7728-7741.

Marchi M, Grilli M (2010) Presynaptic nicotinic receptors modulating neurotransmitter release in the central nervous system: functional interactions with other coexisting receptors. Prog Neurobiol 92:105-111.

Miwa JM, Stevens TR, King SL, Caldarone BJ, Ibanez-Tallon I, Xiao C, Fitzsimonds RM, Pavlides C, Lester HA, Picciotto MR, Heintz N (2006) The prototoxin lynx1 acts on nicotinic acetylcholine receptors to balance neuronal activity and survival in vivo. Neuron 51:587-600.

Miwa JM, Lester HA, Walz A (2012) Optimizing cholinergic tone through lynx modulators of nicotinic receptors: implications for plasticity and nicotine addiction. Physiology (Bethesda) 27:187-199.

Morishita H, Miwa JM, Heintz N, Hensch TK (2010) Lynx1, a cholinergic brake, limits plasticity in adult visual cortex. Science 330:1238-1240.

Nakauchi S, Brennan RJ, Boulter J, Sumikawa K (2007) Nicotine gates longterm potentiation in the hippocampal CA1 region via the activation of alpha2* nicotinic ACh receptors. Eur J Neurosci 25:2666-2681.

Nichols WA, Henderson BJ, Yu C, Parker RL, Richards CI, Lester HA, Miwa JM (2014) Lynx1 shifts $\alpha 4 \beta 2$ nicotinic receptor subunit stoichiometry by affecting assembly in the endoplasmic reticulum. J Biol Chem 289:31423-31432.

Niell CM, Stryker MP (2008) Highly selective receptive fields in mouse visual cortex. J Neurosci 28:7520-7536.

Ochoa V, George AA, Nishi R, Whiteaker P (2016) The prototoxin LYPD6B modulates heteromeric $\alpha 3 \beta 4$-containing nicotinic acetylcholine receptors, but not $\alpha 7$ homomers. FASEB J 30:1109-1119.

Özhan G, Sezgin E, Wehner D, Pfister AS, Kühl SJ, Kagermeier-Schenk B, Kühl M, Schwille P, Weidinger G (2013) Lypd6 enhances Wnt/ $\beta$-catenin signaling by promoting Lrp6 phosphorylation in raft plasma membrane domains. Dev Cell 26:331-345.

Pakan JM, Lowe SC, Dylda E, Keemink SW, Currie SP, Coutts CA, Rochefort NL (2016) Behavioral-state modulation of inhibition is context-dependent and cell type specific in mouse visual cortex. eLife 5:e14985.

Pfeffer CK, Xue M, He M, Huang ZJ, Scanziani M (2013) Inhibition of inhibition in visual cortex: the logic of connections between molecularly distinct interneurons. Nat Neurosci 16:1068-1076.

Polack PO, Friedman J, Golshani P (2013) Cellular mechanisms of brain state-dependent gain modulation in visual cortex. Nat Neurosci 16:13311339.

Porciatti V, Pizzorusso T, Maffei L (1999) The visual physiology of the wild type mouse determined with pattern VEPs. Vision Res 39:3071-3081.

Prusky GT, Douglas RM (2003) Developmental plasticity of mouse visual acuity. Eur J Neurosci 17:167-173.

Puddifoot CA, Wu M, Sung RJ, Joiner WJ (2015) Ly6h regulates trafficking of $\alpha 7$ nicotinic acetylcholine receptors and nicotine-induced potentiation of glutamatergic signaling. J Neurosci 35:3420-3430.

Reimers K, Emmert N, Shah H, Benedict RH, Szigeti K (2014) Capgras-like visual decomposition in Lewy body dementia with therapeutic response to donepezil. Neurol Clin Pract 4:467-469.

Sadahiro M, Sajo M, Morishita H (2016) Nicotinic regulation of experiencedependent plasticity in visual cortex. J Physiol Paris 110:29-36.

Silberberg G, Markram H (2007) Disynaptic inhibition between neocortical pyramidal cells mediated by Martinotti cells. Neuron 53:735-746.

Smith MR, Burman P, Sadahiro M, Kidd BA, Dudley JT, Morishita H (2016) Integrative analysis of disease signatures shows inflammation disrupts juvenile experience-dependent cortical plasticity. eNeuro 3:ENEURO.024016.2016.

Stark E, Eichler R, Roux L, Fujisawa S, Rotstein HG, Buzsáki G (2013) Inhibition-induced theta resonance in cortical circuits. Neuron 80:12631276.

Sturgill JF, Isaacson JS (2015) Somatostatin cells regulate sensory response fidelity via subtractive inhibition in olfactory cortex. Nat Neurosci 18:531535.

Tang Y, Stryker MP, Alvarez-Buylla A, Espinosa JS (2014) Cortical plasticity induced by transplantation of embryonic somatostatin or parvalbumin interneurons. Proc Natl Acad Sci U S A 111:18339-18344.

Tasic B, Menon V, Nguyen TN, Kim TK, Jarsky T, Yao Z, Levi B, Gray LT, Sorensen SA, Dolbeare T, Bertagnolli D, Goldy J, Shapovalova N, Parry S, Lee C, Smith K, Bernard A, Madisen L, Sunkin SM, Hawrylycz M, et al. 
(2016) Adult mouse cortical cell taxonomy revealed by single cell transcriptomics. Nat Neurosci 19:335-346.

Tasic B, Yao Z, Graybuck LT, Smith KA, Nguyen TN, Bertagnolli D, Goldy J, Garren E, Economo MN, Viswanathan S, Penn O, Bakken T, Menon V, Miller J, Fong O, Hirokawa KE, Lathia K, Rimorin C, Tieu M, Larsen R, et al. (2018) Shared and distinct transcriptomic cell types across neocortical areas. Nature 563:72-78.

Thomsen MS, Cinar B, Jensen MM, Lyukmanova EN, Shulepko MA, Tsetlin V, Klein AB, Mikkelsen JD (2014) Expression of the Ly-6 family proteins Lynx1 and Ly6H in the rat brain is compartmentalized, cell-type specific, and developmentally regulated. Brain Struct Funct 219:1923-1934.

Timmermann DB, Sandager-Nielsen K, Dyhring T, Smith M, Jacobsen AM, Nielsen EO, Grunnet M, Christensen JK, Peters D, Kohlhaas K, Olsen GM, Ahring PK (2012) Augmentation of cognitive function by NS9283, a stoichiometry-dependent positive allosteric modulator of $\alpha 2$ - and $\alpha 4$ containing nicotinic acetylcholine receptors. Br J Pharmacol 167:164182.

Urban DJ, Roth BL (2015) DREADDs (designer receptors exclusively activated by designer drugs): chemogenetic tools with therapeutic utility. Annu Rev Pharmacol Toxicol 55:399-417.

van Versendaal D, Rajendran R, Saiepour MH, Klooster J, Smit-Rigter L, Sommeijer JP, De Zeeuw CI, Hofer SB, Heimel JA, Levelt CN (2012)
Elimination of inhibitory synapses is a major component of adult ocular dominance plasticity. Neuron 74:374-383.

Wang J, Kuryatov A, Jin Z, Norleans J, Kamenecka TM, Kenny PJ, Lindstrom J (2015a) A novel $\alpha 2 / \alpha 4$ subtype-selective positive allosteric modulator of nicotinic acetylcholine receptors acting from the C-tail of an $\alpha$ subunit. J Biol Chem 290:28834-28846.

Wang J, Kuryatov A, Sriram A, Jin Z, Kamenecka TM, Kenny PJ, Lindstrom J (2015b) An accessory agonist binding site promotes activation of $\alpha 4 \beta 2^{\star}$ nicotinic acetylcholine receptors. J Biol Chem 290:13907-13918.

Wiesel TN (1982) Postnatal development of the visual cortex and the influence of environment. Nature 299:583-591.

Xu H, Jeong HY, Tremblay R, Rudy B (2013) Neocortical somatostatinexpressing GABAergic interneurons disinhibit the thalamorecipient layer 4. Neuron 77:155-167.

Xu H, Liu L, Tian Y, Wang J, Li J, Zheng J, Zhao H, He M, Xu T-L, Duan S, $\mathrm{Xu} H$ (2019) A disinhibitory microcircuit mediates conditioned social fear in the prefrontal cortex. Neuron 102:668-682.e5.

Yaeger CE, Ringach DL, Trachtenberg JT (2019) Neuromodulatory control of localized dendritic spiking in critical period cortex. Nature 567:100104 .

Zhao-Shea R, Liu L, Pang X, Gardner PD, Tapper AR (2013) Activation of GABAergic neurons in the interpeduncular nucleus triggers physical nicotine withdrawal symptoms. Curr Biol 23:2327-2335. 